\title{
SOME IRREDUCIBLE FREE GROUP REPRESENTATIONS IN WHICH A LINEAR COMBINATION OF THE GENERATORS HAS AN EIGENVALUE
}

\author{
WILLIAM L. PASCHKE \\ (Received 2 August 2000; revised 14 February 2001) \\ Communicated by G. Willis
}

\begin{abstract}
We construct irreducible unitary representations of a finitely generated free group which are weakly contained in the left regular representation and in which a given linear combination of the generators has an eigenvalue. When the eigenvalue is specified, we conjecture that there is only one such representation. The representation we have found is described explicitly (modulo inversion of a certain rational map on Euclidean space) in terms of a positive definite function, and also by means of a quasi-invariant probability measure on the combinatorial boundary of the group.
\end{abstract}

2000 Mathematics subject classification: primary 46L54, $22 \mathrm{D} 25$.

\section{Statement of main result}

Let $G$ be the free group on $n$ generators $u_{1}, u_{2}, \ldots, u_{n}$, where $n \geq 2$. For a reduced word $s$ in $G$, let $|s|_{i}$ for each index $i$ (respectively $\gamma(s)$ ) be the number of occurrences of $u_{i}$ or $u_{i}^{-1}$ (respectively $u_{k}^{-1} u_{j}$ for $k \neq j$ ) in $s$. Given positive numbers $c_{1}, \ldots, c_{n}$ and a positive number $\lambda$ satisfying $2 \max c_{i}^{2}-\sum_{i} c_{i}^{2}<\lambda^{2}<\sum_{i} c_{i}^{2}$, let $\left(x_{1}, \ldots, x_{n}\right)$ be the unique vector in the positive orthant of $\mathbb{R}^{n}$ satisfying

$$
\frac{x_{j}\left(1+\sum_{i \neq j} x_{i}\right)}{\sum_{i} x_{i}}=\frac{c_{j}^{2}}{\lambda^{2}}
$$

for each $j$. Define numbers $a_{1}, \ldots, a_{n}$ by

$$
a_{j}=\sqrt{\frac{x_{j}}{\left(1+\sum_{i \neq j} x_{i}\right) \sum_{i} x_{i}}} .
$$

(C) 2002 Australian Mathematical Society $1446-7887 / 2002 \$ A 2.00+0.00$ 
The function $\phi$ defined on $G$ by $\phi(s)=\left(-\sum_{j} x_{j}\right)^{\gamma(s)} \prod_{j} a_{j}^{|s|_{j}}$ is positive definite and satisfies $\sum_{i} c_{i} \phi\left(s u_{i}\right)=\lambda \phi(s)$ for all $s$ in $G$. The associated unitary representation $\pi$ is irreducible and weakly contained in the left regular representation (so $\phi$ extends to a pure state of the reduced group $C^{*}$-algebra $\left.C_{r}^{*}(G)\right)$. The $\lambda$-eigenspace of $\sum_{i} c_{i} \pi\left(u_{i}\right)$ is one-dimensional.

\section{Introduction}

To make the discussion below flow a bit more smoothly, we will adopt the following terminology. By a state of $G$, we mean a unital positive definite function on $G$. We will often regard a state of $G$ as a positive linear functional on the complex group algebra $C G$, or on the universal $C^{*}$-algebra $C^{*}(G)$, or (if appropriate) on the reduced $C^{*}$-algebra $C_{r}^{*}(G)$, that is, the $C^{*}$-algebra generated by the image of $G$ in its left regular representation on $\ell^{2}(G)$. We call a state reduced if it is bounded with respect to the operator norm on $C G$ in the left regular representation (and so extends to a state of $C_{r}^{*}(G)$ ). In general, we will use the term reduced to mean 'having to do with the left regular representation', so for instance the reduced spectrum of an element in $\mathbb{C} G$ is its spectrum in the left regular representation. We will call a unitary representation of $G$ reduced if it is weakly contained in the left regular representation, that is, if it extends to a $*$-representation of $C_{r}^{*}(G)$. (We will avoid the locution 'reduced irreducible representation', however.) For a complex number $\lambda$ and $X$ in $C G$, a $\lambda$-eigenstate for $X$ is a state $\phi$ of $G$ such that $\phi\left(\left(X^{*}-\bar{\lambda}\right)(X-\lambda)\right)=0$. This is equivalent to $\phi(s(X-\lambda))=0$ for all $s$ in $G$. If $\pi$ is the unitary representation obtained from $\phi$ by the Gel'fand-Raikov construction and $\xi_{0}$ the corresponding distinguished cyclic vector, then clearly $\phi$ is a $\lambda$-eigenstate for $X$ if and only if $\xi_{0}$ is a $\lambda$-eigenvector for $\pi(X)$. Finally, we will call a state of $G$ pure if it is not a proper convex combination of two different states of $G$. This is equivalent to irreducibility of the associated unitary representation.

Consider $Y$ in $C G \backslash\{0\}$. If $Y$ is singular in $C_{r}^{*}(G)$, there is at least one reduced 0 -eigenstate for $Y$; this is because unilateral and bilateral invertibility coincide in $C_{r}^{*}(G)$. We conjectured in $[\mathrm{P}]$ that there are only finitely many reduced pure 0 eigenstates for $Y$, and proved this when $Y=u_{1}+\cdots+u_{n}-\lambda$ with $|\lambda|=\sqrt{n}$ (in which case there is a unique reduced 0 -eigenstate for $Y$ ), and also when $Y$ is a polynomial in one of the generators (where the reduced pure eigenstates correspond to distinct zeros of the polynomial on the unit circle). Kuhn and Steger [KS3], using facts developed in [FTS], have very recently shown that a selfadjoint linear combination $X$ of the generators and their inverses has a unique reduced $\lambda$-eigenstate when $\lambda$ is plus or minus the reduced spectral radius (= reduced norm) of $X$. The results in [P] and [KS3] resemble one another in treating only extreme spectral values, but the techniques of 
proof are utterly different.

At any rate, reduced pure eigenstates for linear combinations of group elements appear to be special enough that there is some point to seeking them out in simple cases with a view to describing them and their associated representations in detail. In what follows, we consider a linear combination $X=c_{1} u_{1}+\cdots+c_{n} u_{n}$ of the generators. The reduced spectrum of $X$ is the closed annulus with outer radius the euclidean length of the coefficient vector $\vec{c}$, and inner radius either zero if no $\left|c_{i}\right|^{2}$ exceeds the sum of the other $\left|c_{j}\right|^{2}$ 's, or else the square root of the largest $\left|c_{i}\right|^{2}$ minus the sum of the other $\left|c_{j}\right|^{2}$ 's. For $\lambda$ in the reduced spectrum, the problem we address is mostly that of finding a reduced $\lambda$-eigenstate for $X$, but at the end of Section 5 we record another marginal uniqueness result. Namely, when $|\lambda|=|\vec{c}|$ the argument in $[\mathrm{P}]$ goes over essentially verbatim to show that there is only one reduced $\lambda$-eigenstate; the case in which $|\lambda|$ is the inner radius of the spectral annulus (provided the latter is positive) then follows easily. For other $\lambda$ in the reduced spectrum, we can only conjecture uniqueness, but it is encouraging that the reduced $\lambda$-eigenstate we identify turns out to be pure, and that the $\lambda$-eigenspace of $X$ in the associated representation turns out to be one-dimensional.

In the case of equal coefficients treated in [P], considerations of symmetry and economy give one a good idea ahead of time of what the formula for a reduced $\lambda$ eigenstate should be. The present case is more suspenseful. We look for the desired needle in two different haystacks - essentially, among $\lambda$-eigenstates which are rarely reduced, and among reduced states which are rarely $\lambda$-eigenstates. The states in the first search venue are defined in terms of the functions $|\cdot|_{i}$ and $\gamma_{i j}$ that count, respectively, the number of $u_{i}$ 's and $u_{i}^{-1}$ 's in a reduced word, and the number of $u_{i}^{-1} u_{j}$ 's and $u_{j}^{-1} u_{i}$ 's. (Thus $\gamma_{i j}=\gamma_{j i}$ and $\gamma_{i i}=0$. Notice also that $\sum_{i}|\cdot|_{i}$ is the usual length function on $G$ corresponding to the given generators.) We assume henceforth that the coefficients $c_{j}$ are all positive (else rotate by scalars of modulus one, and reduce the number of generators if any of the $c$ 's vanish). Write $\vec{c}=\left(c_{1}, c_{2}, \ldots, c_{n}\right)$. Let $B=\left(b_{i j}\right)$ be a positive $n \times n$ matrix with real entries and with 1's on the diagonal. Assume further (mostly for convenience) that $B \vec{c} \cdot \vec{c}>0$. Define the scalar $\lambda$ and the vector $\vec{a}=\left(a_{1}, a_{2}, \ldots, a_{n}\right)$ by $\lambda=\sqrt{B \vec{c} \cdot \vec{c}}$ and $\vec{a}=\lambda^{-1} B \vec{c}$. Define $\phi$ on $G$ by

$$
\phi(s)=\phi_{B, \bar{c}}(s)=\prod_{i} a_{i}^{|s|_{i}-\sum_{j} \gamma_{i j}(s)} \prod_{i>j} b_{i j}^{\gamma_{i j}(s)}
$$

(where we read $0^{0}$ as 1). Thus, $\phi\left(u_{j}\right)=a_{j}, \phi\left(u_{i} u_{j}\right)=a_{i} a_{j}, \phi\left(u_{i} u_{j}^{-1}\right)=a_{i} a_{j}(i \neq j)$, $\phi\left(u_{i}^{-1} u_{j}\right)=b_{i j}(i \neq j)$, and so on. It turns out that $\phi$ satisfies $\sum_{i} c_{i} \phi\left(s u_{i}\right)=\lambda \phi(s)$ for all $s$ in $G$ (easy), is positive definite on $G$ (moderately difficult), and is pure (strenuous), which is to say that the unitary representation of $G$ to which $\phi$ gives rise is irreducible. Our proof of irreducibility relies crucially on a result of Linnell [L] which says that the kernel of a matrix over $\mathbb{C} G$, viewed as a map between direct sums 
of $\ell^{2}(G)$ 's, has integer von Neumann dimension. (Linnell's clever argument for this involves the Fredholm module that is sometimes used in demonstrating the absence of nontrivial idempotents in $C_{r}^{*}(G)$.) We need only the following special case.

THEOREM 2.1. Every nonzero element of $\mathbb{C} G$ has zero kernel in the left regular representation.

The $\lambda$ 's that arise in the present context are the positive numbers in the spectrum of $\sum c_{i} u_{i}$ in the universal representation. The latter is the annulus with outer radius $\sum c_{i}$ and inner radius $\max \left\{0,2 \max c_{i}-\sum c_{i}\right\}$. More restrictively, suppose that $\lambda$ is positive and lies in the interior of the spectrum of $\sum c_{i} u_{i}$ in the left regular representation, that is, $2 \max c_{i}^{2}-\sum c_{i}^{2}<\lambda^{2}<\sum c_{i}^{2}$. There are many eligible matrices $B$ such that $B \vec{c} \cdot \vec{c}=\lambda^{2}$. Which one of these-and we imagine there can be at most one-is such that $\phi_{B, \vec{c}}$ is reduced, that is, extends to a state of $C_{r}^{*}(G)$ ?

At this point, the search moves to the second haystack, namely boundary representations of $G$. To obtain one of these, take the combinatorial boundary $\Omega$ of $G$, consisting of all reduced one-way infinite strings in the $u_{i}$ 's and their inverses, topologize it compactly with cylinder sets $\Omega(s)$ (= set of strings in $\Omega$ beginning with the reduced word $s$ ), and put on $\Omega$ a Borel probability measure that is quasi-invariant under the natural left action of $G$. Let $P_{1}, \ldots, P_{n}$ be measurable functions such that

$$
\left|P_{i}(\omega)\right|^{2}=\frac{d \mu\left(u_{i}^{-1} \omega\right)}{d \mu(\omega)} .
$$

For each $i$, we get a unitary operator $U_{i}$ on $L^{2}(\Omega, \mu)$ by setting

$$
\left(U_{i} f\right)(\omega)=P_{i}(\omega) f\left(u_{i}^{-1} \omega\right),
$$

and hence a unitary representation $\pi$-called a boundary representation-of $G$ on $L^{2}(\Omega, \mu)$. All boundary representations of $G$ are weakly contained in the left regular representation [Spi], so the states obtained by composing them with vector states are all reduced. What is needed for our purposes would seem to be readily available: (1) a probability measure $\mu$ (which can be obtained by prescribing the function $s \mapsto \mu(\Omega(s))$ from $G$ to $[0,1]$ subject to the obvious compatibility requirement), with the Radon-Nikodým derivatives $d \mu \circ u_{i}^{-1} / d \mu$ in good shape; and (2) measurable functions $q_{i}$ of modulus one such that

$$
\sum_{i} c_{i} q_{i}(\omega) \sqrt{\frac{d \mu\left(u_{i}^{-1} \omega\right)}{d \mu(\omega)}}=\lambda
$$

for $\mu$-almost all $\omega$. If $P_{i}=q_{i} \sqrt{d\left(\mu \circ u_{i}^{-1}\right) / d \mu}$, and $\pi$ is the resulting unitary representation of $G$, then the unit constant function 1 is a $\lambda$-eigenvector for $\sum c_{i} \pi\left(u_{i}\right)$, and $\phi=\langle\pi(\cdot) 1,1\rangle$ is a state of the type we are seeking. 
A diligent search, however, finds only one such choice of $\mu$ and associated $q_{i}$ 's (which turn out to be \pm 1 -valued). This apparatus is described in Section 4 below. We show there that $\phi=\langle\pi(\cdot) 1,1\rangle$ is of the form $\phi_{B, \vec{c}}$, where the entries of $B$ are obtained as follows. Let $\mathbb{R}_{+}^{n}$ denote the positive orthant of $\mathbb{R}^{n}$, and let $D_{n}$ be the set of points $\left(s_{1}, \ldots, s_{n}\right)$ in $\mathbb{R}_{+}^{n}$ satisfying $2 \max s_{i}-\sum s_{i}<1<\sum s_{i}$. It is shown in the appendix that the map $S: \mathbb{R}_{+}^{n} \rightarrow D_{n}$ defined by

$$
S\left(x_{1}, \ldots, x_{n}\right)=(1 / t)\left(x_{1}\left(1+y_{1}\right), \ldots, x_{n}\left(1+y_{n}\right)\right),
$$

where

$$
t=\sum_{i} x_{i} \text { and for each } j, y_{j}=\sum_{i \neq j} x_{i},
$$

is bijective. Notice that $\left(c_{1}^{2} / \lambda^{2}, \ldots, c_{n}^{2} / \lambda^{2}\right) \in D_{n}$. Let

$$
\left(x_{1}, \ldots, x_{n}\right)=S^{-1}\left(c_{1}^{2} / \lambda^{2}, \ldots, c_{n}^{2} / \lambda^{2}\right) .
$$

The matrix $B=\left\{\phi\left(u_{i}^{-1} u_{j}\right)\right\}$ has entry

$$
b_{i j}=-\sqrt{\frac{x_{i} x_{j}}{\left(1+y_{i}\right)\left(1+y_{j}\right)}}
$$

for $i \neq j$. The associated $a_{i}$ 's are given by $a_{i}=\sqrt{x_{i} /\left(t\left(1+y_{i}\right)\right)}$. With this particular choice of inputs, the state $\phi_{B, \bar{c}}$ becomes the one described in Section 1 above.

We thank the referee of this paper for numerous helpful suggestions, but above all for supplying the highly efficient topological argument following Proposition 6.3 in the appendix. This permits the bijectivity of the map $S$ described above to be demonstrated with only a modest amount of explicit calculation.

\section{Pure eigenstates}

Fix positive numbers $c_{1}, \ldots, c_{n}$ and a positive (semidefinite) $n \times n$ matrix $B=\left(b_{i j}\right)$ with real entries such that $b_{i i}=1$ for each $i$. With $\vec{c}=\left(c_{1}, \ldots, c_{n}\right)$, let $\lambda=\sqrt{B \vec{c} \cdot \vec{c}}$. We assume henceforth that $\lambda>0$. Let $\vec{a}=\lambda^{-1} B \vec{c}$. Define $\phi: G \rightarrow \mathbb{R}$ by

$$
\phi(s)=\prod_{i} a_{i}^{|s|_{i}-\sum_{j} \gamma_{i j}(s)} \prod_{i>j} b_{i j}^{\gamma_{i j}(s)},
$$

where $\mid \cdot l_{i}$ (respectively $\gamma_{i j}$ ) counts the number of occurrences of $u_{i}$ or $u_{i}^{-1}$ (respectively $u_{i}^{-1} u_{j}$ or $u_{j}^{-1} u_{i}$ ) in a reduced word in $G$. We will show in this section that $\phi$ is a pure $\lambda$-eigenstate of $G$ for $\sum_{i} c_{i} u_{i}$.

The algebraic properties of $\phi$ are easily established. 
LEMMA 3.1. (a) The function $\phi$ satisfies:

$\phi(1)=1$

$\phi(s)=\phi\left(s^{-1}\right)$ for all $s$ in $G$;

$\phi\left(u_{i} s\right)=a_{i} \phi(s)$ for $s$ in $G$ not beginning with $u_{i}^{-1}$;

$\phi\left(s u_{i}\right)=a_{i} \phi(s)$ for $s$ in $G$ not ending in the inverse of a generator;

$\phi\left(s u_{j}^{-1} u_{i}\right)=b_{i j} \phi(s)$ for $s$ not ending in $u_{j}$.

(b) The properties in part (a) characterize $\phi$ among real functions on $G$.

(c) For all $s$ in $G$, we have $\sum_{i} c_{i} \phi\left(s u_{i}\right)=\lambda \phi(s)$.

PROOF. (a) All of the ingredients of the definition are unchanged when $s$ is replaced by $s^{-1}$. The $\gamma$ 's and all of the $|\cdot|$ 's except for $|\cdot|_{i}$ are unchanged when $s$ not beginning with $u_{i}^{-1}$ is multiplied on the left by $u_{i}$. The same is true for right multiplication by $u_{i}$ provided $s$ does not end in the inverse of a generator. If $i \neq j$ and $s$ does not end in $u_{j}$, then right multiplication of $s$ by $u_{j}^{-1} u_{i}$ increases $\gamma_{i j}, \gamma_{j i},|\cdot|_{j}$, and $|\cdot|_{i}$ by 1 , and leaves all other $\gamma$ 's and $|\cdot|$ 's unchanged. Thus the exponent of $b_{i j}=b_{j i}$ in the formula for $\phi$ increases by 1 , and all other exponents in the formula are unchanged.

(b) The properties in (a) imply that $\phi\left(u_{i}^{ \pm 1}\right)=a_{i}$, and furthermore permit calculation of $\phi$ on words of a given length from its values on words of smaller length.

(c) If $s$ does not end in the inverse of a generator, then

$$
\sum_{i} c_{i} \phi\left(s u_{i}\right)=\left(\sum_{i} a_{i} c_{i}\right) \phi(s)=\frac{B \vec{c} \cdot \vec{c}}{\lambda} \phi(s)=\lambda \phi(s) .
$$

Otherwise, $s=t u_{j}^{-1}$ for some $t$ not ending in $u_{j}$ and we have

$$
\sum_{i \neq j} c_{i} \phi\left(s u_{i}\right)+c_{j} \phi\left(s u_{j}\right)=\left(\sum_{i \neq j} b_{j i} c_{i}+c_{j}\right) \phi(t)=\lambda a_{j} \phi(t)=\lambda \phi(s),
$$

where we have used $b_{j j}=1, B \vec{c}=\lambda \vec{a}$, and $\phi(s)=\phi\left(s^{-1}\right)=\phi\left(u_{j} t^{-1}\right)=a_{j} \phi(t)$.

To begin the construction of the representation that has $\phi$ as a matrix entry, let $G^{+}$ be the unital semigroup in $G$ generated by $u_{1}, \ldots, u_{n}$, and let $G_{k}^{+}$be the set of group elements in $G^{+}$of length $k$. For $k=1,2, \ldots$, let $A_{k}$ be the $n^{k} \times n^{k}$ matrix with entries indexed by $G_{k}^{+} \times G_{k}^{+}$whose $(s, t)$-entry is $\phi\left(s^{-1} t\right)$.

LEMMA 3.2. The matrix $A_{k}$ is positive for $k=1,2, \ldots$

PROOF. Notice that $A_{1}=B$. For the inductive step, regard $G_{k+1}^{+}$as the disjoint union of $n$ copies of $G_{k}^{+}$by writing $G_{k+1}^{+}=u_{1} G_{k}^{+} \cup u_{2} G_{k}^{+} \cup \cdots \cup u_{n} G_{k}^{+}$. We can then write $A_{k+1}$ in terms of $A_{k}$ as an $n \times n$ matrix of $n^{k} \times n^{k}$ matrices. For $s, t$ in $G_{k}^{+}$, we have

$$
\phi\left(s^{-1} u_{i}^{-1} u_{j} t\right)= \begin{cases}\phi\left(s^{-1} t\right), & i=j \\ b_{i j} \phi(s) \phi(t), & i \neq j\end{cases}
$$


Thus the $i, j$ entry of $A_{k+1}$, viewed in this way, is $A_{k}$ if $i=j$ and $b_{i j} X_{k}$ if $i \neq j$, where $X_{p}$, for a positive integer $p$, is the matrix of the (positive, one-dimensional) operator on $\ell^{2}\left(G_{p}^{+}\right)$defined by $\left(X_{p} \vec{\xi}\right)(s)=\left(\sum_{t \in G_{p}^{+}} \xi(t) \phi(t)\right) \phi(s)$. Notice that $X_{1} \vec{\xi}=(\vec{\xi} \cdot \vec{a}) \vec{a}$ for $\vec{\xi}$ in $\ell^{2}\left(G_{1}^{+}\right)$. We may write

$$
A_{k+1}=A_{k} \otimes I+X_{k} \otimes(B-I)=\left(A_{k}-X_{k}\right) \otimes I+X_{k} \otimes B .
$$

in tensor products of $G_{k}^{+} \times G_{k}^{+}$matrices and $n \times n$ matrices. Now for $s$ in $G^{+}$, we have $\phi\left(u_{i} s\right)=a_{i} \phi(s)$, whence it follows that $X_{k+1}=X_{k} \otimes X_{1}$. This means that

$$
A_{k+1}-X_{k+1}=\left(A_{k}-X_{k}\right) \otimes I+X_{k} \otimes\left(B-X_{1}\right) .
$$

Once we show that $B-X_{1}$ is positive, it will follow by induction that $A_{k}-X_{k}$, and hence $A_{k}$, is positive for all $k$. For $\vec{\xi}=\left(\xi_{1}, \ldots, \xi_{n}\right)$ in $\ell^{2}\left(G_{1}^{+}\right)$, we have

$$
X_{1} \vec{\xi} \cdot \vec{\xi}=|\vec{\xi} \cdot \vec{a}|^{2}=\frac{|\vec{\xi} \cdot B \vec{c}|^{2}}{\lambda^{2}} \leq \frac{(B \vec{\xi} \cdot \vec{\xi})(B \vec{c} \cdot \vec{c})}{\lambda^{2}}=B \vec{\xi} \cdot \vec{\xi},
$$

showing $B-X_{1} \geq 0$ as promised.

The Hilbert space $H$ of the representation we seek is constructed as follows. By Lemma 3.2, there is for each positive integer $k$ a finite dimensional complex inner product space $E_{k}$ spanned by vectors $\left\{\Delta_{s}: s \in G_{k}^{+}\right\}$with inner product $\langle\cdot, \cdot\rangle$ satisfying $\left\langle\Delta_{t}, \Delta_{s}\right\rangle=\phi\left(s^{-1} t\right)$. (We write $E_{0}$ for the one-dimensional inner product space spanned by the unit vector $\Delta_{1}$.) Because

$$
\lambda^{-2} \sum_{i, j} c_{i} c_{j} \phi\left(u_{j}^{-1} t^{-1} s u_{i}\right)=\phi\left(t^{-1} s\right)
$$

for all $s, t$ in $G^{+}$, we have an isometry from $E_{k}$ into $E_{k+1}$ for each $k$ sending $\Delta_{s}$, for $s$ in $G_{k}^{+}$, to $\lambda^{-1} \sum_{i} c_{i} \Delta_{s u_{i}}$. Let $H_{0}$ be the Hilbert space inductive limit of the resulting tower $E_{0} \rightarrow E_{1} \rightarrow E_{2} \rightarrow \cdots$. Then $H_{0}$ is the closed linear span of $\left\{\Delta_{s}: s \in G^{+}\right\}$, and these vectors satisfy

$$
\sum_{i} c_{i} \Delta_{s u_{i}}=\lambda \Delta_{s} \quad \text { and } \quad\left\langle\Delta_{t}, \Delta_{s}\right\rangle=\phi\left(s^{-1} t\right) .
$$

Left multiplication by each generator $u_{i}$ gives rise to an isometry $V_{i}$ of $H_{0}$ into itself. Let $H_{i}^{\prime}=H_{0} \ominus V_{i} H_{0}$. For each $i$, let $S_{i}^{-}$be the subset of $G$ consisting of the reduced words ending in $u_{i}^{-1}$. The Hilbert space $H$ is

$$
H=H_{0} \oplus \bigoplus_{i=1}^{n}\left(\ell^{2}\left(S_{i}^{-}\right) \otimes H_{i}^{\prime}\right) .
$$


For each $i$, let $U_{i}$ be the unitary operator on $H$ that maps $H_{0}$ to $V_{i} H_{0}$ by $V_{i}$, maps $\delta_{u_{i}^{-1}} \otimes H_{i}^{\prime}$ to $H_{i}^{\prime}=H_{0} \ominus V_{i} H_{0}$ by erasing the tensor, and maps $\delta_{s} \otimes \eta$ to $\delta_{u_{i} s} \otimes \eta$ for all other $s$ ending in the inverse of a generator, and for $\eta$ in the appropriate space $H_{j}^{\prime}$. Denote by $\pi$ the unitary representation of $G$ on $H$ that takes $u_{i}$ to $U_{i}$. Our next goal is to show that $\phi=\left\langle\pi(\cdot) \Delta_{1}, \Delta_{1}\right\rangle$.

Write $P_{i}$ for the orthogonal projection of $H_{0}$ on $H_{i}^{\prime}$.

LEMMA 3.3. (a) $P_{i} \Delta_{1}=\Delta_{1}-a_{i} \Delta_{u_{i}}$ and $U_{i}^{*} \Delta_{1}=\delta_{u_{i}^{-1}} \otimes P_{i} \Delta_{1}+a_{i} \Delta_{1}$.

(b) For $s$ in $u_{j} G^{+}$and $i \neq j$, we have $P_{i} \Delta_{s}=\Delta_{s}-\phi\left(u_{i}^{-1} s\right) \Delta_{u_{i}}$ and

$$
U_{i}^{*} \Delta_{s}=\delta_{u_{i}^{-1}} \otimes P_{i} \Delta_{s}+\phi\left(u_{i}^{-1} s\right) \Delta_{1} .
$$

(c) For $\eta \in U_{j} H_{0}$ and $i \neq j$, we have $\left(1-P_{i}\right) \eta=\left\langle\eta, \Delta_{u_{i}}\right\rangle \Delta_{u_{i}}$.

PROOF. (a) Both statements follow from the observation that

$$
\left(\Delta_{u_{i} l}, \Delta_{1}-a_{i} \Delta_{u_{i}}\right)=\phi\left(u_{i} t\right)-a_{i} \phi(t)=0 \text { for all } t \text { in } G^{+} .
$$

(b) Notice that $\left\langle\Delta_{s}-\phi\left(u_{i}^{-1} s\right) \Delta_{u_{i}}, \Delta_{u_{i} t}\right\rangle=\phi\left(t^{-1} u_{i}^{-1} s\right)-\phi\left(t^{-1}\right) \phi\left(u_{i}^{-1} s\right)=0$ for all $t$ in $G^{+}$because there is no cancellation in $u_{i}^{-1} s$.

(c) The $\Delta_{s}$ 's with $s$ as in (b) span a dense subspace of $U_{j} H_{0}$, so this follows from (b).

LEMMA 3.4. For each $t$ in $G$, the vector $\pi(t) \Delta_{1}$ may be written in the form $\xi+h \Delta_{w}$, where $h$ is a real number, $w \in G^{+}$, and $\xi$ is either zero or a finite sum of terms $\delta_{r} \otimes \eta_{r}$, where each $r$ belongs to some $S_{i}^{-}$and begins with the same generator or inverse generator as $t$, and $\eta_{r}$ belongs to the corresponding $H_{i}^{\prime}$. Furthermore, $w=1$ if $t$ begins with the inverse of a generator.

PROOF. This is obvious if $t \in G^{+}$(the case in which $\xi=0$ ). By what we have observed just above, $t=u_{i}^{-1}$ makes $\xi=\delta_{u_{i}^{-1}} \otimes P_{i} \Delta_{i}, h=a_{i}$, and $w=1$. For $s$ in $u_{j} G^{+}$and $i \neq j$, we also have $\pi\left(u_{i}^{-1} s\right) \Delta_{1}=\delta_{u_{i}^{-1}} \otimes P_{i} \Delta_{s}+\phi\left(u_{i}^{-1} s\right) \Delta_{1}$, and furthermore $\pi\left(u_{k}^{-1} u_{i}^{-1} s\right) \Delta_{1}=\delta_{u_{k}^{-1} u_{i}^{-1}} \otimes P_{i} \Delta_{s}+\phi\left(u_{i}^{-1} s\right)\left(\delta_{u_{k}^{-1}} \otimes P_{k} \Delta_{1}+a_{k} \Delta_{1}\right)$, while $\pi\left(u_{k} u_{i}^{-1} s\right) \Delta_{1}=\delta_{u_{k} u_{i}^{-1}} \otimes P_{i} \Delta_{s}+\phi\left(u_{i}^{-1} s\right) \Delta_{u_{k}}$ when $k \neq i$. And so forth-the asserted form plainly remains intact under further noncancelling left multiplication by generators or inverse generators.

PROPOSITION 3.5. For all $t$ in $G$, we have $\left\langle\pi(t) \Delta_{1}, \Delta_{1}\right\rangle=\phi(t)$.

ProOF. Write $\pi(t) \Delta_{1}=\xi+h \Delta_{w}$ as in Lemma 3.4. If $t$ does not begin with $u_{i}^{-1}$, then $U_{i} \xi \perp H_{0}$ and so

$$
\left\langle\pi\left(u_{i} t\right) \Delta_{1}, \Delta_{1}\right\rangle=h\left(\Delta_{u_{i} w}, \Delta_{1}\right)=h a_{i} \phi(w)=a_{i}\left\langle\pi(t) \Delta_{1}, \Delta_{1}\right\rangle .
$$


Furthermore, for $j \neq i$ we have $U_{j}^{*} U_{i} \xi \perp H_{0}$ and

$$
\left\langle\pi\left(u_{j}^{-1} u_{i} t\right) \Delta_{1}, \Delta_{1}\right\rangle=h\left\langle\Delta_{u_{i} w}, \Delta_{u_{j}}\right\rangle=h \phi\left(u_{j}^{-1} u_{i} w\right)=b_{i j} h \phi(w)=b_{i j}\left(\pi(t) \Delta_{1}, \Delta_{1}\right\rangle .
$$

If $t$ begins with $u_{i}^{-1}$, then $w=1$, and for any $j$

$$
\left\langle\pi\left(u_{j}^{-1} t\right) \Delta_{1}, \Delta_{1}\right\rangle=\left\langle\pi(t) \Delta_{1}, \Delta_{u_{j}}\right\rangle=h\left\langle\Delta_{1}, \Delta_{u_{j}}\right\rangle=a_{j} h=a_{j}\left\langle\pi(t) \Delta_{1}, \Delta_{1}\right\rangle .
$$

Notice also that $\left\langle\pi(t) \Delta_{1}, \Delta_{1}\right\rangle=\left\langle\pi\left(t^{-1}\right) \Delta_{1}, \Delta_{1}\right\rangle$ (because $h$ is real). It now follows from part (b) of Lemma 3.1 that $\left\langle\pi(\cdot) \Delta_{1}, \Delta_{1}\right\rangle$ coincides with $\phi$.

\section{THEOREM 3.6. The representation $\pi$ is irreducible.}

PROOF. We begin by noticing that $\Delta_{1}$ is cyclic for $\pi$. Indeed, by Lemma 3.3, the linear span of $\pi(G) \Delta_{1}$ contains $\delta_{u_{i}^{-1}} \otimes P_{i} \Delta_{s}$ for each $s$ in $G^{+}$and each $i$. It follows that the closed linear span contains each summand $\ell^{2}\left(S_{i}^{-}\right) \otimes H_{i}^{\prime}$. Of course, it also contains $H_{0}$ because $\pi(s) \Delta_{1}=\Delta_{s}$ for $s$ in $G^{+}$.

We will show in several steps that the commutant $\pi(G)^{\prime}$ of $\pi(G)$ consists of scalars. Our argument makes essential use of Theorem 2.1 above.

Claim 1. If $T \in \pi(G)^{\prime}$, then $T \Delta_{1}$ is orthogonal to $\ell^{2}\left(S_{i}^{-}\right) \otimes P_{i} \Delta_{1}$ for each $i$.

Proof OF Claim 1. Since $\Delta_{1}$ is a $\lambda$-eigenvector for $\sum_{i} c_{i} \pi\left(u_{i}\right)$, so is $T \Delta_{1}$. Thus

$$
\begin{aligned}
\lambda\left\langle T \Delta_{1}, \Delta_{1}\right\rangle & =\sum_{i} c_{i}\left\langle T \Delta_{1}, U_{i}^{*} \Delta_{1}\right\rangle=\sum_{i} c_{i}\left\langle T \Delta_{1}, \delta_{u_{i}^{-1}} \otimes P_{i} \Delta_{1}+a_{i} \Delta_{1}\right\rangle \\
& =\lambda\left\langle T \Delta_{1}, \Delta_{1}\right\rangle+\sum_{i} c_{i}\left\langle T \Delta_{1}, \delta_{u_{i}^{-1}} \otimes P_{i} \Delta_{1}\right\rangle,
\end{aligned}
$$

where we have used Lemma 3.3 and the identity $\sum_{i} a_{i} c_{i}=\lambda$. This makes

$$
\sum_{i} c_{i}\left\langle T \Delta_{1}, \delta_{u_{i}^{-1}} \otimes P_{i} \Delta_{1}\right\rangle=0
$$

Define $\xi$ on $G$ by setting $\xi(t)=\left\langle T \Delta_{1}, \delta_{t} \otimes P_{i} \Delta_{1}\right\rangle$ if $t \in S_{i}^{-}$for some $i$, and $\xi(t)=0$ for all other $t$ in $G$. Then $\sum_{j} c_{j} \xi\left(u_{j}^{-1} t\right)=\lambda \xi(t)$

(a) for $t$ in $\bigcup_{i} S_{i}^{-}$because $T \Delta_{1}$ is a $\lambda$-eigenvector for $\sum_{j} c_{j} \pi\left(u_{j}\right)$;

(b) for $t=1$ by ( $*$ ) above, because $\xi(1)=0$; and

(c) for all other $t$ in $G$ because for such $t$ all of the values of $\xi$ in the asserted relationship are zero.

Because the vectors $\delta_{t} \otimes P_{i} \Delta_{1}$ that appear in the nonzero part of the definition of $\xi$ are mutually orthogonal and norm-bounded, the function $\xi$ belongs to $\ell^{2}(G)$. The claim now follows from Theorem 2.1. 
ClaIM 2. For $T$ in $\pi(G)^{\prime}$ and $s$ in $G^{+}$, we have $\left\langle T \Delta_{1}, \Delta_{u_{i}}\right\rangle=a_{i}\left\langle T \Delta_{1}, \Delta_{s}\right\rangle$ for each $i$, and hence $\left\langle T \Delta_{1}, \Delta_{s}\right\rangle=\phi(s)\left\langle T \Delta_{1}, \Delta_{1}\right\rangle$ for all $s$ in $G^{+}$.

ProOF OF ClaIM 2. We see using Lemma 3.3 and Claim 1 applied to $T^{*}$ that

$$
\begin{aligned}
\left\langle T \Delta_{1}, \Delta_{u_{i} s}\right\rangle & =\left\langle\delta_{u_{i}^{-1}} \otimes P_{i} \Delta_{1}+a_{i} \Delta_{1}, \pi(s) T^{*} \Delta_{1}\right\rangle \\
& =\left\langle\delta_{s^{-1} u_{i}^{-1}} \otimes P_{i} \Delta_{1}, T^{*} \Delta_{1}\right\rangle+a_{i}\left\langle\Delta_{1}, T^{*} \Delta_{s}\right\rangle=a_{i}\left\langle T \Delta_{1}, \Delta_{s}\right\rangle .
\end{aligned}
$$

The second assertion follows by induction on the length of $s$.

Claim 3. $T \Delta_{1} \in H_{0}$ for $T$ in $\pi(G)^{\prime}$.

PROOF OF ClaiM 3. It will suffice to show that

$$
T \Delta_{1} \perp \ell^{2}\left(S_{j}^{-}\right) \otimes P_{j} \Delta_{u_{i} s}
$$

for $j \neq i$ and $s$ in $G^{+}$. We proceed more or less as in the proof of Claim 1. Fix $s$ in $G^{+}$and the index $i$. Define $\xi$ on $G$ by setting $\xi(t)=\left\langle T \Delta_{1}, \delta_{t} \otimes P_{j} \Delta_{u_{i}}\right\rangle$ if $t \in S_{j}^{-}$ for some $j \neq i$, and $\xi(t)=0$ for all other $t$ in $G$. Thus $\xi \in \ell^{2}(G)$. As in the proof of Claim 1, we will show that $\xi$ must vanish by showing that it satisfies

$$
\sum_{k} c_{k} \xi\left(u_{k}^{-1} t\right)=\lambda \xi(t)
$$

for all $t$ in $G$. This identity holds if $t \in S_{i}^{-}$or if $t$ ends in a generator because all of the values of $\xi$ that appear are zero. It holds if $t \in S_{j}^{-}$for some $j \neq i$ because $T \Delta_{1}$ is a $\lambda$-eigenvector for $\sum_{k} c_{k} \pi\left(u_{k}\right)$. The only remaining case is $t=1$, for which we must show that $\sum_{k \neq i} c_{k} \xi\left(u_{k}^{-1}\right)=0$. But

$$
\begin{aligned}
\lambda a_{i} \phi(s)\left\langle T \Delta_{1}, \Delta_{1}\right\rangle & =\lambda\left\langle T \Delta_{1}, \Delta_{u_{i} s}\right\rangle=\sum_{k} c_{k}\left\langle T \Delta_{1}, U_{k}^{*} \Delta_{u_{i} s}\right\rangle \\
& =c_{i}\left\langle T \Delta_{1}, \Delta_{s}\right\rangle+\sum_{k \neq i} c_{k}\left\langle T \Delta_{1}, \delta_{u_{k}^{-1}} \otimes P_{k} \Delta_{u_{i} s}+b_{i k} \phi(s) \Delta_{1}\right\rangle \\
& =c_{i} \phi(s)\left\langle T \Delta_{1}, \Delta_{1}\right\rangle+\sum_{k \neq i} b_{i k} c_{k} \phi(s)\left\langle T \Delta_{1}, \Delta_{1}\right\rangle+\sum_{k \neq i} c_{k} \xi\left(u_{k}^{-1}\right) \\
& =\lambda a_{i} \phi(s)\left\langle T \Delta_{1}, \Delta_{1}\right\rangle+\sum_{k \neq i} c_{k} \xi\left(u_{k}^{-1}\right),
\end{aligned}
$$

where we have used Claim 2, Lemma 3.3 above, and $B \vec{c}=\lambda \vec{a}$.

It follows from Claim 2 that $T \Delta_{1}-\left\langle T \Delta_{1}, \Delta_{1}\right\rangle \Delta_{1}$ is orthogonal to $\Delta_{s}$ for every $s$ in $G^{+}$, and hence $T \Delta_{1}=\left\langle T \Delta_{1}, \Delta_{1}\right\rangle \Delta_{1}$ by Claim 3 , for all $T$ in $\pi(G)^{\prime}$. Because $\Delta_{1}$ is cyclic for $\pi$, this proves that $\pi$ is irreducible. 


\section{Boundary representations}

The representations considered in this section are all weakly contained in the left regular representation, so we must restrict attention to $\lambda$ 's in the spectrum of $\sum c_{i} u_{i}$ in that representation.

PROPOSITION 4.1. Let complex coefficients $c_{1}, c_{2}, \ldots, c_{n}$ be given, and let $X=$ $\sum c_{i} u_{i}$. The reduced spectrum of $X$ is

$$
\left\{\lambda \in \mathbb{C}: r_{0} \leq|\lambda| \leq\left(\sum\left|c_{i}\right|^{2}\right)^{1 / 2}\right\}
$$

where $r_{0}^{2}$ is zero if none of the $\left|c_{i}\right|^{2}$ 's exceeds the sum of the others, and is otherwise the maximum of the $\left|c_{i}\right|^{2}$ 's minus the sum of the others.

PROOF. The spectrum is connected because the reduced $C^{*}$-algebra of $G$ contains no nontrivial idempotents [PV]. It is rotationally invariant because there is an automorphism of this $C^{*}$-algebra that multiplies each $u_{i}$ by a given scalar of modulus one. Thus, the spectrum must be either a closed disc about 0 or a closed annulus. Let $\|\cdot\|_{\text {op }}$ denote the reduced operator norm. Then for every positive integer $k$ we have

$$
\left\|X^{k}\right\|_{2} \leq\left\|X^{k}\right\|_{\text {op }} \leq(k+1)\left\|X^{k}\right\|_{2}
$$

where the lower bound is obvious and the upper bound follows from Haagerup's inequality $[\mathrm{H}]$ plus the observation that $X^{k}$ is a linear combination of words of length $k$. Since the coefficient of a $k$-fold product of generators in $X^{k}$ is the corresponding product of $c$ 's, we have

so

$$
\left\|X^{k}\right\|_{2}=\|X\|_{2}^{k}=\left(\sum\left|c_{i}\right|^{2}\right)^{k / 2},
$$

$$
\left(\sum\left|c_{i}\right|^{2}\right)^{1 / 2} \leq\left\|X^{k}\right\|_{\mathrm{op}}^{1 / k} \leq(k+1)^{1 / k}\left(\sum\left|c_{i}\right|^{2}\right)^{1 / 2}
$$

This shows that the reduced spectral radius of $X$ is the 2-norm of the coefficient vector. It remains to show that we have correctly identified the inner radius $r_{0}$, which we do by induction on the number of nonzero coefficients. In the case of only one nonzero coefficient, say $c_{1}$, the spectrum is the circle about 0 of radius $\left|c_{1}\right|$, which plainly coincides with $r_{0}$. Suppose that the proposition gives the correct inner radius when there are $m-1$ nonzero coefficients. Without loss of generality, we may assume $X=c_{1} u_{1}+\cdots+c_{m-1} u_{m-1}+u_{m}$, where $\left|c_{1}\right|, \ldots,\left|c_{m-1}\right| \leq 1$. Let $Y=\left(c_{1} u_{1}+\cdots c_{m-1} u_{m-1}\right) u_{m}^{-1}$. Notice that $u_{1} u_{m}^{-1}, \ldots, u_{m-1} u_{m}^{-1}$ and $u_{m}^{-1}$ constitute a set of free generators for $G$. There are two cases: (1) $\left|c_{1}\right|^{2}+\cdots+\left|c_{m-1}\right|^{2} \geq 1$; and 
(2) the contrary. In case (1), the inner radius of the reduced spectrum of $Y$ is at most 1 by our induction hypothesis (plus the assumption that the $|c|$ 's are all at most 1 ) and the outer radius is at least 1 by the spectral radius formula already established. Thus $X\left(=(Y+1) u_{m}\right)$ is not invertible in the left regular representation, giving $r_{0}=0$ as required in this case. In case (2), let

$$
r=\sqrt{1-\left|c_{1}\right|^{2}-\cdots-\left|c_{m-1}\right|^{2}} .
$$

If $|\lambda|<r$, then the reduced spectral radius of $Y-\lambda u_{m}^{-1}$ is less than 1 , and hence $X-\lambda\left(=\left(Y-\lambda u_{m}^{-1}+1\right) u_{m}\right)$ is invertible in the left regular representation. This shows that the reduced spectrum of $X$ has inner radius at least $r$. On the other hand, $X-r$ is singular in the left regular representation because $Y-r u_{m}^{-1}$ has reduced spectral radius precisely 1 . This shows that the reduced spectrum of $X$ has the correct inner radius in case (2), completing the proof.

We briefly review some essential facts about the combinatorial boundary of $G$. Let $V$ be the set $\left\{u_{1}, u_{1}^{-1}, u_{2}, u_{2}^{-1}, \ldots, u_{n}, u_{n}^{-1}\right\}$. The combinatorial boundary $\Omega$ of $G$ is the set of all infinite strings $\omega=v_{1} v_{2} \ldots$ where each $v_{i} \in V$ and $v_{i+1} \neq v_{i}^{-1}$ for all $i$. It becomes a compact Hausdorff space when equipped with the product topology, a basis for which consists of cylinder sets described as follows. For each positive integer $k$, let $p_{k}$ be the map from $\Omega$ to $G$ that reads the first $k$ symbols. For $s$ in $G$ with $|s|=k$, let $\Omega(s)=p_{k}^{-1}(\{s\})$, that is, the set of strings in $\Omega$ beginning with $s$. Also let $\Omega(1)=\Omega$. The $\Omega(s)$ 's are all open and closed, and form a basis for the topology of $\Omega$. For each $s$ in $G$, let $G(s)$ be the set of reduced words in $G$ beginning with $s$, with $G(1)=G$. Further let $E_{s}$ be the orthogonal projection of $\ell^{2}(G)$ on $\ell^{2}(G(s))$, and let $A$ be the $C^{*}$-algebra generated by the $E_{s}$ 's-that is, the norm closure of their linear span. Then $A$ is commutative, and $A$ modulo its intersection with the ideal $K$ of compact operators is easily seen to be isomorphic to $C(\Omega)$. (Let $\phi$ be a multiplicative linear functional on $A$ that kills $A \cap K$. Since each $E_{s}$ is a one-dimensional operator plus the sum of $E_{s v}$ over $v$ in $V$ such that $s v$ is reduced, there is a unique $\omega$ in $\Omega$ such that $\phi\left(E_{p_{m}(\omega)}\right)=1$ for all $m$. On the other hand, given $\omega$ in $\Omega$, one obtains a multiplicative linear functional on $A$ by taking a weak* limit of the vector states $\left\langle\cdot \delta_{p_{k}(\omega)}, \delta_{p_{k}(\omega)}\right\rangle$. In this way, one has a continuous bijection between $\Omega$ and the maximal ideal space of $A /(A \cap K)$.) Borel measures on $\Omega$ are determined by their values on cylinder sets, and can be defined by prescribing those values subject only to an obvious compatibility condition. In fact, given any function $\hat{\mu}: G \rightarrow[0,1]$ with $\hat{\mu}(1)=1$ such that

$$
\hat{\mu}(s)=\sum\{\hat{\mu}(s v): v \in V,|s v|=|s|+1\}
$$

for every $s$ in $G$, there is a Borel probability measure $\mu$ such that $\mu(\Omega(s))=\hat{\mu}(s)$ for all $s$. (To see this, notice that for every $k$, the sum of the values of $\hat{\mu}$ on the words of 
length $k$ is 1 . Let $\xi_{k}$ be $\sqrt{\hat{\mu}}$ times the indicator function of the set of words of length $k$, so $\xi_{k}$ is a unit vector in $\ell^{2}(G)$. Any weak ${ }^{*}$-limit of the corresponding vector states on $A$ gives the desired $\mu$, because $\left\langle E_{s} \xi_{k}, \xi_{k}\right\rangle=\hat{\mu}(s)$ for $k \geq|s|$.) We will continue to use the notation $\hat{\mu}$ for the function on $G$ corresponding to the measure $\mu$ on $\Omega$.

One type of eligible $\hat{\mu}$ can be specified by choosing functions $\beta: V \rightarrow[0,1]$ and $\alpha: V \times V \rightarrow[0,1]$ such that

$$
\sum_{v \in V} \beta(v)=1, \quad \sum_{v^{\prime} \in V} \alpha\left(v, v^{\prime}\right)=1, \quad \alpha\left(v, v^{-1}\right)=0 \quad \forall v \in V,
$$

and then setting $\hat{\mu}(1)=1, \hat{\mu}(v)=\beta(v)$ for $v$ in $V$, and for reduced words of length two or greater, $\hat{\mu}\left(v_{1} v_{2} \cdots v_{k}\right)=\beta\left(v_{1}\right) \alpha\left(v_{1}, v_{2}\right) \cdots \alpha\left(v_{k-1}, v_{k}\right)$. (In other words, the measure $\mu$ is the probability measure on the space of sample paths in a Markov chain with states labeled by $V$, transition probabilities given by $\alpha$, and initial probabilities given by $\beta$.) If all of the values of $\beta$ and of $\alpha$ (except the $\alpha\left(v, v^{-1}\right)$ 's) are positive, the measure $\mu$ is quasi-invariant under the natural left action of $G$ on $\Omega$, and the Radon-Nikodým derivatives of the translates of $\mu$ by the generators of $G$ are easily calculated. Namely we have

$$
\frac{d \mu\left(u_{i}^{-1} \omega\right)}{d \mu(\omega)}= \begin{cases}\frac{\beta(v)}{\beta\left(u_{i}\right) \alpha\left(u_{i}, v\right)}, & p_{2}(\omega)=u_{i} v ; \\ \frac{\beta\left(u_{i}^{-1}\right) \alpha\left(u_{i}^{-1}, v\right)}{\beta(v)}, & p_{1}(\omega)=v \neq u_{i},\end{cases}
$$

because for reduced words $s$ of length at least 2 , the ratio $\mu\left(u_{i}^{-1} \Omega(s)\right) / \mu(\Omega(s))$ depends only on the first two symbols in $s$ (in the manner indicated by the switches in the formula).

Let positive numbers $c_{1}, c_{2}, \ldots, c_{n}$ be given, as well as a positive number $\lambda$ satisfying $\lambda^{2}<\sum_{i} c_{i}^{2}$ and $c_{j}^{2}-\sum_{i \neq j} c_{i}^{2}<\lambda^{2}$ for $j=1, \ldots, n$. Our immediate aim is to exhibit a Borel probability measure $\mu$ on $\Omega$ and a unitary representation of $G$ on $L^{2}(\Omega, \mu)$ in which $c_{1} u_{1}+c_{2} u_{2}+\cdots+c_{n} u_{n}$ has $\lambda$ as an eigenvalue. This can be done fairly cleanly in terms of the inverse of the map $S$ defined on the positive orthant of $\mathbb{R}^{n}$ by

$$
S\left(x_{1}, x_{2}, \ldots, x_{n}\right)=\left(\frac{x_{j}\left(1+\sum_{i \neq j} x_{i}\right)}{\sum_{i} x_{i}}\right)_{j=1}^{n} .
$$

It is shown in the appendix that $S$ is injective on the positive orthant. Furthermore, the conditions we have imposed on $\lambda$ ensure that $\lambda^{-2}\left(c_{1}^{2}, c_{2}^{2}, \ldots, c_{n}^{2}\right)$ belongs to the range of $S$. Accordingly, we write

$$
\left(x_{1}, x_{2}, \ldots, x_{n}\right)=S^{-1}\left(\frac{c_{1}^{2}}{\lambda^{2}}, \frac{c_{2}^{2}}{\lambda^{2}}, \ldots, \frac{c_{n}^{2}}{\lambda^{2}}\right) .
$$


As in the appendix, we write $t=\sum_{i} x_{i}$ and $y_{j}=t-x_{j}=\sum_{i \neq j} x_{i}$. The measure $\mu$ is the one constructed as in the previous paragraph for $\beta$ and $\alpha$ defined by

$$
\begin{array}{ll}
\beta\left(u_{i}\right)=\frac{x_{i}}{t(1+t)}, & \alpha\left(u_{j}, u_{i}\right)=\alpha\left(u_{j}^{-1}, u_{i}^{-1}\right)=\frac{x_{i}}{t\left(1+y_{j}\right)}, \\
\beta\left(u_{i}^{-1}\right)=\frac{x_{i}}{1+t}, & \alpha\left(u_{j}, u_{i}^{-1}\right)=\alpha\left(u_{j}^{-1}, u_{i}\right)=\frac{x_{i}}{1+y_{j}}, \quad i \neq j,
\end{array}
$$

and $\alpha\left(u_{i}, u_{i}^{-1}\right)=0=\alpha\left(u_{i}^{-1}, u_{i}\right)$. It is readily checked that $\alpha$ and $\beta$ satisfy all of the sum-to- 1 conditions of the previous paragraph. Radon-Nikodým derivatives under translation by the generators are given by

$$
\frac{d \mu\left(u_{i}^{-1} \omega\right)}{d \mu(\omega)}= \begin{cases}t\left(1+y_{i}\right) / x_{i}, & p_{1}(\omega)=u_{i} \\ t x_{i} /\left(1+y_{i}\right), & p_{1}(\omega)=u_{j}, j \neq i \\ x_{i} / t\left(1+y_{i}\right), & p_{1}(\omega)=u_{j}^{-1} .\end{cases}
$$

Define $P:\left\{u_{1}, u_{2}, \ldots, u_{n}\right\} \times \Omega \rightarrow \mathbb{R}$ by

$$
P\left(u_{i}, \omega\right)= \begin{cases}\sqrt{t\left(1+y_{i}\right) / x_{i}}, & p_{1}(\omega)=u_{i} \\ -\sqrt{t x_{i} /\left(1+y_{i}\right)}, & p_{1}(\omega)=u_{j}, j \neq i \\ \sqrt{x_{i} / t\left(1+y_{i}\right)}, & p_{1}(\omega)=u_{j}^{-1}\end{cases}
$$

so $P\left(u_{i}, \omega\right)^{2}=d \mu\left(u_{i}^{-1} \omega\right) / d \mu(\omega)$. Define unitaries $U_{1}, U_{2}, \ldots, U_{n}$ on $L^{2}(\Omega, \mu)$ by $\left(U_{i} \xi\right)(\omega)=P\left(u_{i}, \omega\right) \xi\left(u_{i}^{-1} \omega\right)$, and let $\pi$ be the unitary representation of $G$ on this Hilbert space that sends each $u_{i}$ to the corresponding $U_{i}$. It follows from [Spi, Theorem 2.7] (see also [KS1, Theorem IX]) that $\pi$ is weakly contained in the left regular representation.

PROPOSITION 4.2. We have $\sum_{i} c_{i} \pi\left(u_{i}\right) 1=\lambda 1$, where 1 is the unit constant function on $\Omega$.

ProOF. Since $\pi\left(u_{i}\right) 1=P\left(u_{i}, \cdot\right)$, this amounts to showing that

$$
\sum_{i} \frac{c_{i}}{\lambda} P\left(u_{i}, \omega\right)=1
$$

for all $\omega$. By construction, $c_{i} / \lambda=\sqrt{x_{i}\left(1+y_{i}\right) / t}$ for each $i$. If $\omega \in \Omega\left(u_{j}^{-1}\right)$ for some $j$, we have

$$
\sum_{i} \frac{c_{i}}{\lambda} P\left(u_{i}, \omega\right)=\sum_{i} \sqrt{\frac{x_{i}\left(1+y_{i}\right)}{t}} \sqrt{\frac{x_{i}}{t\left(1+y_{i}\right)}}=\sum_{i} \frac{x_{i}}{t}=1 .
$$


Otherwise, $\omega \in \Omega\left(u_{j}\right)$ for some $j$, and we have

$$
\begin{aligned}
\sum_{i} \frac{c_{i}}{\lambda} P\left(u_{i}, \omega\right) & =-\sum_{i \neq j} \sqrt{\frac{x_{i}\left(1+y_{i}\right)}{t}} \sqrt{\frac{t x_{i}}{1+y_{i}}}+\sqrt{\frac{x_{j}\left(1+y_{j}\right)}{t}} \sqrt{\frac{t\left(1+y_{j}\right)}{x_{j}}} \\
& =-\sum_{i \neq j} x_{i}+1+y_{j}=1 .
\end{aligned}
$$

We now take up the project of identifying the reduced state $\langle\pi(\cdot) 1,1\rangle$. Let us call this state $\phi$. To begin with, $\phi$ can be expressed in terms of the cocycle $P$ (with values in the multiplicative group of nonzero reals) on $G \times \Omega$ that extends the function $P$ defined above. Thus $(\pi(s) \xi)(\omega)=P(s, \omega) \xi\left(s^{-1} \omega\right)$ for $s$ in $G$ and $\omega$ in $\Omega$. In particular, $(\pi(s) 1)(\omega)=P(s, \omega)$ and so $\phi(s)=\int_{\Omega} P(s, \omega) d \mu(\omega)$. The cocycle identity satisfied by $P$ is $P(r s, \omega)=P(r, \omega) P\left(s, r^{-1} \omega\right)$. Since $P(1, \omega)=1$, we have in particular

$$
P\left(u_{i}^{-1}, \omega\right)=\frac{1}{P\left(u_{i}, u_{i} \omega\right)}= \begin{cases}\sqrt{x_{i} / t\left(1+y_{i}\right)}, & p_{1}(\omega) \neq u_{i}^{-1} \\ -\sqrt{\left(1+y_{i}\right) / t x_{i}}, & p_{2}(\omega)=u_{i}^{-1} u_{j} \\ \sqrt{t\left(1+y_{i}\right) / x_{i}}, & p_{2}(\omega)=u_{i}^{-1} u_{j}^{-1}\end{cases}
$$

It also follows from the cocycle identity that for an arbitrary reduced word $v_{1} v_{2} \cdots v_{k}$ with each $v_{i}$ in $V$ we may write $P\left(v_{1} v_{2} \cdots v_{k}, \omega\right)$ as

$$
P\left(v_{1}, \omega\right) P\left(v_{2}, v_{1}^{-1} \omega\right) P\left(v_{3}, v_{2}^{-1} v_{1}^{-1} \omega\right) \cdots P\left(v_{k}, v_{k-1}^{-1} \cdots v_{2}^{-1} v_{1}^{-1} \omega\right) .
$$

This formula leads to the following useful observation.

LEMMA 4.3. (a) If the reduced word $r$ begins with $u_{i}^{-1}$ for some $i$, then $P(r, \cdot)$ is constant on $\Omega\left(u_{1}\right) \cup \Omega\left(u_{2}\right) \cup \cdots \cup \Omega\left(u_{n}\right)$.

(b) On the other hand, $P(r, \cdot)$ is constant on $\Omega\left(u_{i}^{-1}\right)$ provided $r$ does not begin with $u_{i}^{-1}$.

PROOF. (a) Write $\Omega(+)=\Omega\left(u_{1}\right) \cup \Omega\left(u_{2}\right) \cup \cdots \cup \Omega\left(u_{n}\right)$, and $r=u_{i}^{-1} v_{2} \cdots v_{k}$. Observe that $P\left(u_{i}^{-1}, \cdot\right)$ is constant on $\Omega(+)$, in fact on $\Omega \backslash \Omega\left(u_{i}^{-1}\right)$. Whether $v_{2}$ is $u_{j}$ for some $j \neq i$ or $u_{j}^{-1}$, the factor $P\left(v_{2}, u_{i}(\cdot)\right)$ in the formula is constant on $\Omega(+)$; notice that $u_{i} \Omega(+) \subseteq \Omega\left(u_{i}\right)$. The remaining factors in the formula for $P(r, \cdot)$ are constant on $\Omega(+)$ because for $v$ in $V$, the function $P(v, \cdot)$ reads at most only the first two symbols in its argument.

(b) Suppose $r=u_{j}^{-1} v_{2} \cdots v_{k}$ for some $j \neq i$ (and hence $v_{2} \neq u_{j}$ ). Observe that $P\left(u_{j}^{-1}, \cdot\right)$ is constant on $\Omega\left(u_{i}^{-1}\right)$, in fact on $\Omega \backslash \Omega\left(u_{j}^{-1}\right)$. We have $u_{j} \Omega\left(u_{i}^{-1}\right) \subseteq$ $\Omega\left(u_{j} u_{i}^{-1}\right)$, so $P\left(v_{2}, u_{j}(\cdot)\right)$ is constant on $\Omega\left(u_{i}^{-1}\right)$, and the remaining factors are constant there for the same reason as in part (a). Likewise if $r=u_{j} v_{2} \cdots v_{k}$ (and hence $\left.v_{2} \neq u_{j}^{-1}\right)$, the two initial factors $P\left(u_{j}, \cdot\right)$ and $P\left(v_{2}, u_{j}^{-1}(\cdot)\right)$ are constant on $\Omega\left(u_{i}^{-1}\right)$. The remaining factors are constant on $\Omega\left(u_{i}^{-1}\right)$ for the same reason as in part (a). 
We can now undertake the calculation that exhibits $\phi$ as one of the states investigated in Section 3.

PROPOSITION 4.4. For each $i$ and each $j$ different from $i$, let

Then

$$
a_{i}=\sqrt{\frac{x_{i}}{t\left(1+y_{i}\right)}} \text { and } \quad b_{i j}=-\sqrt{\frac{x_{i} x_{j}}{\left(1+y_{i}\right)\left(1+y_{j}\right)}} .
$$

$$
\phi(s)=\prod_{i} a_{i}^{|s|_{i}-\sum_{j} \gamma_{i j}(s)} \prod_{i\rangle j} b_{i j}^{\gamma_{i j}(s)}
$$

where $|\cdot|_{i}$ (respectively $\gamma_{i j}$ ) counts the number of occurrences of $u_{i}$ or $u_{i}^{-1}$ (respectively $u_{i}^{-1} u_{j}$ or $u_{j}^{-1} u_{i}$ ) in a reduced word in $G$.

PROOF. The calculation is in three parts.

1. We show first that if $j \neq i$, and $s$ is a reduced word not beginning with $u_{i}^{-1}$ (including the possibility that $s=1$ ), then $\phi\left(u_{j}^{-1} u_{i} s\right)=b_{i j} \phi(s)$. We have

$$
\begin{aligned}
\phi\left(u_{j}^{-1} u_{i} s\right) & =\left\langle\pi(s) 1, \pi\left(u_{i}^{-1} u_{j}\right) 1\right\rangle=\int_{\Omega} P(s, \omega) \frac{P\left(u_{j}, u_{i} \omega\right)}{P\left(u_{i}, u_{i} \omega\right)} d \mu(\omega) \\
& =\int_{\Omega \backslash \Omega\left(u_{i}^{-1}\right)}+\int_{\Omega\left(u_{i}^{-1} u_{j}\right)}+\sum_{k \neq i, j} \int_{\Omega\left(u_{i}^{-1} u_{k}\right)}+\sum_{k} \int_{\Omega\left(u_{i}^{-1} u_{k}^{-1}\right)} P(s, \omega) \frac{P\left(u_{j}, u_{i} \omega\right)}{P\left(u_{i}, u_{i} \omega\right)} d \mu(\omega) .
\end{aligned}
$$

By Lemma 4.3 (b), there is a number $C$ such that $P(s, \omega)=C$ for all $\omega$ in $\Omega\left(u_{i}^{-1}\right)$. Taking this into account, and looking up values for $P\left(u_{i}, \cdot\right)$ and $P\left(u_{j}, \cdot\right)$ in the various cases that arise, we obtain

$$
\begin{aligned}
\phi\left(u_{j}^{-1} u_{i} s\right)= & -\sqrt{\frac{t x_{j}}{1+y_{j}}} \sqrt{\frac{x_{i}}{t\left(1+y_{i}\right)}} \int_{\Omega \backslash \Omega\left(u_{i}^{-1}\right)} P(s, \omega) d \mu(\omega) \\
& -C \sqrt{\frac{t\left(1+y_{j}\right)}{x_{j}}} \sqrt{\frac{1+y_{i}}{t x_{i}}} \hat{\mu}\left(u_{i}^{-1} u_{j}\right)+C \sum_{k \neq i, j} \sqrt{\frac{t x_{j}}{1+y_{j}}} \sqrt{\frac{1+y_{i}}{t x_{i}}} \hat{\mu}\left(u_{i}^{-1} u_{k}\right) \\
& +C \sum_{k} \sqrt{\frac{x_{j}}{t\left(1+y_{j}\right)}} \sqrt{\frac{t\left(1+y_{i}\right)}{x_{i}}} \hat{\mu}\left(u_{i}^{-1} u_{k}^{-1}\right) .
\end{aligned}
$$

Using $\hat{\mu}\left(u_{i}^{-1} u_{k}^{ \pm 1}\right)=\beta\left(u_{i}^{-1}\right) \alpha\left(u_{i}^{-1}, u_{k}^{ \pm 1}\right)$, this makes

$$
\phi\left(u_{j}^{-1} u_{i} s\right)=b_{i j} \int_{\Omega \backslash \Omega\left(u_{i}^{-1}\right)} P(s, \omega) d \mu(\omega)+C\left(-\sqrt{\frac{\left(1+y_{j}\right)\left(1+y_{i}\right)}{x_{j} x_{i}}} \frac{x_{i}}{1+t} \frac{x_{j}}{1+y_{i}}\right.
$$




$$
\begin{aligned}
& \left.+\sqrt{\frac{x_{j}\left(1+y_{i}\right)}{\left(1+y_{j}\right) x_{i}}} \frac{x_{i}}{1+t} \sum_{k \neq i, j} \frac{x_{k}}{1+y_{i}}+\sqrt{\frac{x_{j}\left(1+y_{i}\right)}{\left(1+y_{j}\right) x_{i}}} \frac{x_{i}}{1+t} \sum_{k} \frac{x_{k}}{t\left(1+y_{i}\right)}\right) \\
= & b_{i j} \int_{\Omega \backslash \Omega\left(u_{i}^{-1}\right)} P(s, \omega) d \mu(\omega) \\
& +\frac{C}{1+t}\left[\left(b_{i j}\left(1+y_{j}\right)-\left(t-x_{i}-x_{j}\right) b_{i j}-b_{i j}\right] .\right.
\end{aligned}
$$

Since $\mu\left(\Omega\left(u_{i}^{-1}\right)\right)=x_{i} /(1+t)$, the second term is simply the $b_{i j}$ times the integral of $P(s, \cdot)$ over $\Omega\left(u_{i}^{-1}\right)$. We are done with the first part of the proof.

2. Next we show that $\phi\left(u_{i} s\right)=a_{i} \phi(s)$ if $s$ is a reduced word not beginning with $u_{i}^{-1}$ (including the possibility $s=1$ ). To begin with,

$$
\begin{aligned}
\phi\left(u_{i} s\right) & =\left\langle\pi(s) 1, \pi\left(u_{i}^{-1}\right) 1\right\rangle=\int_{\Omega} P(s, \omega) P\left(u_{i}^{-1}, \omega\right) d \mu(\omega) \\
& =\int_{\Omega \backslash \Omega\left(u_{i}^{-1}\right)}+\sum_{j \neq i} \int_{\Omega\left(u_{i}^{-1} u_{j}\right)}+\sum_{j} \int_{\Omega\left(u_{i}^{-1} u_{j}^{-1}\right)} P(s, \omega) P\left(u_{i}^{-1}, \omega\right) d \mu(\omega) .
\end{aligned}
$$

Use Lemma 4.3 (b) to find $C$ such that $P(s, \omega)=C$ for $\omega$ in $\Omega\left(u_{i}^{-1}\right)$. Looking up values for $P\left(u_{i}^{-1}, \omega\right)$ in the various cases, we obtain

$$
\begin{aligned}
\phi\left(u_{i} s\right)= & \sqrt{\frac{x_{i}}{t\left(1+y_{i}\right)}} \int_{\Omega \backslash \Omega\left(u_{i}^{-1}\right)} P(s, \omega) d \mu(\omega) \\
& +C\left(-\sqrt{\frac{1+y_{i}}{t x_{i}}} \sum_{j \neq i} \hat{\mu}\left(u_{i}^{-1} u_{j}\right)+\sqrt{\frac{t\left(1+y_{i}\right)}{x_{i}}} \sum_{j} \hat{\mu}\left(u_{i}^{-1} u_{j}^{-1}\right)\right) .
\end{aligned}
$$

Evaluating the $\hat{\mu}$ 's gives

$$
\begin{aligned}
\phi\left(u_{i} s\right)= & a_{i} \int_{\Omega \backslash \Omega\left(u_{i}^{-1}\right)} P(s, \omega) d \mu(\omega) \\
& +C\left(-\sqrt{\frac{1+y_{i}}{t x_{i}}} \frac{x_{i}}{1+t} \sum_{j \neq i} \frac{x_{j}}{1+y_{i}}+\sqrt{\frac{t\left(1+y_{i}\right)}{x_{i}}} \frac{x_{i}}{1+t} \sum_{j} \frac{x_{j}}{\left(1+y_{i}\right) t}\right) \\
= & a_{i} \int_{\Omega \backslash \Omega\left(u_{i}^{-1}\right)} P(s, \omega) d \mu(\omega)+\frac{C a_{i}}{1+t}\left(-y_{i}+t\right) \\
= & a_{i}\left(\int_{\Omega \backslash \Omega\left(u_{i}^{-1}\right)}+\int_{\Omega\left(u_{i}^{-1}\right)} P(s, \omega) d \mu(\omega)\right),
\end{aligned}
$$

and we are done with the second part of the proof. 
3. Finally we show that $\phi\left(s u_{i}\right)=a_{i} \phi(s)$ if $s$ is either 1 or a reduced word ending with $u_{j}$ for some $j$. Let $\Omega(-)=\Omega\left(u_{1}^{-1}\right) \cup \Omega\left(u_{2}^{-1}\right) \cup \cdots \cup \Omega\left(u_{n}^{-1}\right)$. Then

$$
\begin{aligned}
\phi\left(s u_{i}\right) & =\int_{\Omega} P\left(u_{i}, \omega\right) P\left(s^{-1}, \omega\right) d \mu(\omega) \\
& =\int_{\Omega\left(u_{i}\right)}+\sum_{k \neq i} \int_{\Omega\left(u_{k}\right)}+\int_{\Omega(-)} P\left(u_{i}, \omega\right) P\left(s^{-1}, \omega\right) d \mu(\omega) .
\end{aligned}
$$

By Lemma 4.3 (a), we have a $C$ such that $P\left(s^{-1}, \omega\right)=C$ in the first two integrals. Thus

$$
\begin{aligned}
\phi\left(s u_{i}\right)= & C\left(\sqrt{\frac{t\left(1+y_{i}\right)}{x_{i}}} \hat{\mu}\left(u_{i}\right)-\sqrt{\frac{t x_{i}}{1+y_{i}}} \sum_{k \neq i} \hat{\mu}\left(u_{k}\right)\right)+a_{i} \int_{\Omega(-)} P\left(s^{-1}, \omega\right) d \mu(\omega) \\
= & C\left(\sqrt{\frac{t\left(1+y_{i}\right)}{x_{i}}} \frac{x_{i}}{t(1+t)}-\sqrt{\frac{t x_{i}}{1+y_{i}}} \sum_{k \neq i} \frac{x_{k}}{t(1+t)}\right) \\
& +a_{i} \int_{\Omega(-)} P\left(s^{-1}, \omega\right) d \mu(\omega) \\
= & \frac{C a_{i}}{1+t}\left(\left(1+y_{i}\right)-y_{i}\right)+a_{i} \int_{\Omega(-)} P\left(s^{-1}, \omega\right) d \mu(\omega) \\
= & a_{i}\left(\int_{\Omega\left(u_{1}\right) \cup \cdots \cup \Omega\left(u_{n}\right)}+\int_{\Omega(-)} P\left(s^{-1}, \omega\right) d \mu(\omega)\right)=a_{i} \phi\left(s^{-1}\right)=a_{i} \phi(s)
\end{aligned}
$$

since $\mu\left(\Omega\left(u_{1}\right) \cup \cdots \cup \Omega\left(u_{n}\right)\right)=\sum_{k} \beta\left(u_{k}\right)=1 /(1+t)$.

As we observed in Lemma 3.1, parts 1, 2, and 3 above suffice to establish the asserted formula for $\phi$.

We remark that the $a$ 's and $b$ 's above are related as in Section 3. Set $b_{i i}=1$ for each $i$, and let $B$ be the $n \times n$ matrix $\left(b_{i j}\right)$. Then $B$ is positive because $\phi$ is positive definite and $b_{i j}=\phi\left(u_{i}^{-1} u_{j}\right)$. Since $c_{i} / \lambda=\sqrt{x_{i}\left(1+y_{i}\right) / t}$, the $i$ th entry of $B \vec{c} / \lambda$ is

$$
\begin{aligned}
\sqrt{\frac{x_{i}\left(1+y_{i}\right)}{t}}+\sum_{j \neq i} b_{i j} \sqrt{\frac{x_{j}\left(1+y_{j}\right)}{t}} & =\sqrt{\frac{x_{i}\left(1+y_{i}\right)}{t}}-\sqrt{\frac{x_{i}}{t\left(1+y_{i}\right)}} \sum_{j \neq i} x_{j} \\
& =\sqrt{\frac{x_{i}}{t\left(1+y_{i}\right)}}\left(1+y_{i}-y_{i}\right)=a_{i},
\end{aligned}
$$

and $B \vec{c} \cdot \vec{c}=\lambda \sum_{i} a_{i} c_{i}=\lambda \sum_{i} c_{i} \phi\left(u_{i}\right)=\lambda^{2}$ by Proposition 4.2. We thus have the ingredients for one of the states examined in Section 3 , and our $\phi$ is $\phi_{B . \bar{c}}$ for the $B$ we have described.

We can now record (most of) the main result of this paper; see also Proposition 5.5 below. 
THEOREM 4.5. Given positive numbers $c_{1}, c_{2}, \ldots, c_{n}$ and $\lambda$ satisfying

$$
\lambda^{2}<\sum_{i} c_{i}^{2} \text { and } c_{j}^{2}-\sum_{i \neq j} c_{i}^{2}<\lambda^{2} \quad \text { for } j=1, \ldots, n,
$$

there are unique positive numbers $x_{1}, x_{2}, \ldots, x_{n}$ such that

$$
\frac{x_{j}\left(1+\sum_{i \neq j} x_{i}\right)}{\sum_{i} x_{i}}=\frac{c_{j}^{2}}{\lambda^{2}}
$$

for each $j$. The function $\phi$ defined on $G$ by

$$
\phi(s)=\left(-\sum_{j} x_{j}\right)^{\gamma(s)} \prod_{i}\left(\frac{x_{i}}{\left(1+\sum_{j \neq i} x_{j}\right) \sum_{j} x_{j}}\right)^{|s|_{i} / 2},
$$

where $|s|_{i}$ and $\gamma(s)$ are respectively the number of occurrences in $s$ of $u_{i}^{ \pm 1}$ and $u_{j}^{-1} u_{k}$ for $k \neq j$, is a reduced pure $\lambda$-eigenstate for $\sum_{i} c_{i} u_{i}$.

Proof. See the appendix for the existence and uniqueness of the $x_{i}$ 's. The formula for $\phi$ follows from Proposition 4.4 above and the observation that the $a$ 's and $b$ 's there satisfy $b_{i j}=-t a_{i} a_{j}$ for $i \neq j$. (Notice as well that $\gamma=\sum_{j>k} \gamma_{j k}$.) Proposition 4.2 implies that $\phi$ is a $\lambda$-eigenstate for $\sum_{i} c_{i} u_{i}$. That $\phi$ is pure follows from results in Section 3 and our identification of $\phi$ as $\phi_{B, \vec{c}}$ above. Finally, it follows from [Spi, Theorem 2.7] that $\phi$ is reduced.

\section{Further observations}

Somewhat surprisingly, the constant function 1 turns out not to be cyclic for the boundary representation $\pi$ described above. Let $H_{1}$ be the closed linear span of $\pi(G) 1$, so by Proposition 4.4, the restriction $\pi_{1}$ of $\pi$ to $H_{1}$ is one of the irreducible representations described in Section 3 above. After some preparation, we will show that the restriction $\pi_{2}$ of $\pi$ to the orthogonal complement $H_{2}$ of $H_{1}$ is unitarily equivalent to $\pi_{1} \circ \sigma$, where $\sigma$ is the automorphism of $G$ that sends each $u_{i}$ to $u_{i}^{-1}$, and that the latter is unitarily inequivalent to $\pi_{1}$. This behaviour distinguishes $\pi_{1}$ sharply from the representations studied by Kuhn and Steger in [KS1]. It seems likely that $\pi_{1}$ belongs to the 'odd' category in the tripartite classification of irreducible representations proposed and conjectured to be exhaustive in [KS2], but this is a matter that will have to be pursued elsewhere.

Write $\Omega( \pm)=\bigcup_{j=1}^{n} \Omega\left(u_{j}^{ \pm 1}\right)$ as above, and consider $h=\sqrt{t} \chi_{\Omega(+)}-(1 / \sqrt{t}) \chi_{\Omega(-)}$, where the $\chi$ 's are indicator functions. This is a unit vector in $L^{2}(\Omega, \mu)$ because the measures of $\Omega(+)$ and $\Omega(-)$ are respectively $1 /(1+t)$ and $t /(1+t)$. We will see below that $H_{2}$ is the closed $\pi(G)$-invariant subspace generated by $h$. 
LEMMA 5.1. For $v$ in $V$ and for $s$ in $G$ not ending in $v^{-1}$, we have

$$
P\left(v, s^{-1} \omega\right)=\phi(s v) / \phi(s)
$$

either for all $\omega$ in $\Omega(+)$ or for all $\omega$ in $\Omega(-)$.

Proof. When $s=1$, this is immediate from the descriptions just before and after Proposition 4.2 above for $P\left(u_{i}^{ \pm 1}, \cdot\right)$. Suppose $s$ begins with $u_{j}^{ \pm 1}$, so there is no canceling in $s^{-1} \omega$ for $\omega$ in $\Omega(\mp)$. If $s$ doesn't end in $u_{i}$, then

$$
P\left(u_{i}^{-1}, s^{-1} \omega\right)=a_{i}=\phi\left(s u_{i}^{-1}\right) / \phi(s)
$$

for all $\omega$ in $\Omega(\mp)$. This takes care of the case $v=u_{i}^{-1}$. If $s$ ends in $u_{k}$ for some $k$, then $P\left(u_{i}, s^{-1} \omega\right)=a_{i}=\phi\left(s u_{i}\right) / \phi(s)$ for all $\omega$ in $\Omega(\mp)$, while if $s$ ends in $u_{k}^{-1}$ for some $k$ different from $i$, we have $P\left(u_{i}, s^{-1} \omega\right)=-t a_{i}=b_{k i} / a_{k}=\phi\left(s u_{i}\right) / \phi(s)$ for all $\omega$ in $\Omega(\mp)$, which finishes the case $v=u_{i}$.

LEMMA 5.2. The vector $h$ is orthogonal to $\pi(G) 1$.

PROOF. We must show that

$$
\int_{\Omega(-)} P(s, \omega) d \mu(\omega)=t \int_{\Omega(+)} P(s, \omega) d \mu(\omega)
$$

for all $s$ in $G$. This is immediate when $s=1$, since $\mu\left(\Omega\left(u_{j}^{-1}\right)\right)=t \mu\left(\Omega\left(u_{j}\right)\right)$ for each $j$. Suppose that $(\star)$ holds for some given $s$ in $G$ and that $v$ in $V$ is such that $s v$ is reduced. We have

$$
\int_{\Omega(-)}+\int_{\Omega(+)} P(s v, \omega) d \mu(\omega)=\phi(s v)=\frac{\phi(s v)}{\phi(s)}\left(\int_{\Omega(-)}+\int_{\Omega(+)} P(s, \omega) d \mu(\omega)\right)
$$

because $\phi(s)$ is the integral of $P(s, \cdot)$ over $\Omega$. It follows from Lemma 5.1 and $P(s v, \omega)=P(s, \omega) P\left(v, s^{-1} \omega\right)$ that either the $\Omega(-)$ summands above are equal, or the $\Omega(+)$ summands are. Hence both are equal, and $(\star)$ for $s v$ follows from $(\star)$ for $s$ by multiplying by $\phi(s v) / \phi(s)$.

We will use the following lemma (which likely holds in much greater generality) in proving the inequivalence of $\pi_{1}$ and $\pi_{2}$.

LEMMA 5.3. Let $Y$ be a linear combination of 1 and $u_{1}, \ldots, u_{n}$ for which there exists $f$ in $\ell^{2}(G)$ such that $Y * f=\delta_{1}$. Then $Y$ is invertible in the left regular representation. 
PROOF. Write $Y=d_{0}+\sum_{i} d_{i} u_{i}$, where without loss of generality the $d$ 's are all nonzero. Since $d_{0} f(1)+\sum_{i} d_{i} f\left(u_{i}^{-1}\right)=1$, at least one of $f(1), f\left(u_{1}^{-1}\right), \ldots, f\left(u_{n}^{-1}\right)$ must be different from zero. Suppose that $f(1) \neq 0$. For each index $j$, let $g_{j}$ be $f$ times the indicator function of $S_{j}^{-}$(the set of reduced words ending in $u_{j}^{-1}$ ). One checks readily that $Y * g_{j}=d_{j} f\left(u_{j}^{-1}\right) \delta_{1}$. Thus, $g_{j}=d_{j} f\left(u_{j}^{-1}\right) f$, by Theorem 2.1. Since $g_{j}(1)=0 \neq f(1)$, this makes $g_{j}=0$; that is, $f$ vanishes on each $S_{j}^{-}$. Now fix distinct indices $j$ and $k$, and let $r(s)=f\left(s u_{k}\right)$ if $s$ in $S_{j}^{-}$and 0 otherwise. As with the $g_{j}$ 's, we have $Y * r=d_{j} r\left(u_{j}^{-1}\right) \delta_{1}$, so $r$ must be a multiple of $f$, but $r(1)=0$, so $r=0$. Continuing in this fashion, we see that $f$ must be supported on $G^{+}$, and it then follows by equating coefficients that $f(1)=1 / d_{0}, f\left(u_{j}\right)=-d_{j} / d_{0}^{2}$, and in general $f\left(u_{i_{1}} u_{i_{2}} \cdots u_{i_{m}}\right)=(-1)^{m} d_{i_{1}} d_{i_{2}} \cdots d_{i_{m}} / d_{0}^{m+1}$. This makes

$$
\left|d_{0}\right|^{2}\|f\|_{2}^{2}=\sum_{m=0}^{\infty}\left(\frac{\left|d_{1}\right|^{2}+\cdots+\left|d_{n}\right|^{2}}{\left|d_{0}\right|^{2}}\right)^{m},
$$

so $\left|d_{1}\right|^{2}+\cdots+\left|d_{n}\right|^{2}<\left|d_{0}\right|^{2}$, so $Y$ is invertible by Proposition 4.1.

If $f(1)=0$, then $f\left(u_{j}^{-1}\right) \neq 0$ for some $j$. We have

$$
\left(d_{j}+d_{0} u_{j}^{-1}+\sum_{i \neq j, 0} d_{i} u_{i} u_{j}^{-1}\right) *\left(u_{j} * f\right)=\delta_{1},
$$

and $\left(u_{j} * f\right)(1)=f\left(u_{j}^{-1}\right)$, so we may apply the previous case to the free generators $u_{j}^{-1}, u_{i} u_{j}^{-1}(i \neq j)$.

PROPOSITION 5.4. (a) The subspace $H_{2}$ is the closed linear span of $\pi(G) h$.

(b) The representation $\pi_{2}$ is unitarily equivalent to $\pi_{1} \circ \sigma$, where $\sigma$ is the automorphism of $G$ sending each $u_{i}$ to $u_{i}^{-1}$.

(c) The representations $\pi_{1}$ and $\pi_{2}$ are unitarily inequivalent.

Proof. Consider the symmetry $T: \Omega \rightarrow \Omega$ taking each string in $\Omega$ to the string obtained by inverting each symbol. It is immediate that $T(\Omega(s))=\Omega(\sigma(s))$. Since

$$
\frac{\hat{\mu}\left(\sigma\left(v_{1} v_{2} \cdots v_{k}\right)\right)}{\hat{\mu}\left(v_{1} v_{2} \cdots v_{k}\right)}=\frac{\beta\left(v_{1}^{-1}\right) \alpha\left(v_{1}^{-1}, v_{2}^{-1}\right) \cdots \alpha\left(v_{k-1}^{-1}, v_{k}^{-1}\right)}{\beta\left(v_{1}\right) \alpha\left(v_{1}, v_{2}\right) \cdots \alpha\left(v_{k-1}, v_{k}\right)}=\frac{\beta\left(v_{1}^{-1}\right)}{\beta\left(v_{1}\right)}
$$

for a nonempty reduced word $v_{1} v_{2} \cdots v_{k}$ with the $v$ 's in $V$, it follows that

$$
\frac{d \mu(T \omega)}{d \mu(\omega)}= \begin{cases}t, & \omega \in \Omega(+) ; \\ 1 / t, & \omega \in \Omega(-),\end{cases}
$$

so $h^{2}=d(\mu \circ T) / d \mu$. The operator $W$ on $L^{2}(\Omega, \mu)$ defined by

$$
(W f)(\omega)=h(\omega) f(T \omega)
$$


is unitary and takes 1 to $h$. We claim that $W$ intertwines $\pi$ and $\pi \circ \sigma$. For $f$ in $L^{2}(\Omega, \mu)$, we have

$$
\left(W \pi\left(u_{i}\right) f\right)(\omega)=h(\omega) P\left(u_{i}, T \omega\right) f\left(u_{i}^{-1} T \omega\right),
$$

while

$$
\left(\pi\left(u_{i}^{-1}\right) W f\right)(\omega)=h\left(u_{i} \omega\right) P\left(u_{i}^{-1}, \omega\right) f\left(u_{i}^{-1} T \omega\right) .
$$

It is readily checked that $h(\omega) P\left(u_{i}, T \omega\right)$ and $h\left(u_{i} \omega\right) P\left(u_{i}^{-1}, \omega\right)$ are the same for all $\omega$, namely

$$
\begin{cases}-1 /\left(\sqrt{t} a_{i}\right), & \omega \in \Omega\left(u_{i}^{-1}\right) \\ \sqrt{t} a_{i}, & \omega \in \Omega \backslash \Omega\left(u_{i}^{-1}\right) .\end{cases}
$$

(One must check two subcases in each of the two cases above.) The intertwining of each $\pi\left(u_{i}\right)$ with its inverse, and thus of $\pi$ with $\pi \circ \sigma$, now follows.

Now let $K$ be the closed $\pi(G)$-invariant subspace of $L^{2}(\Omega, \mu)$ generated by 1 and $h$. Parts (a) and (b) will both follow once we show that $K=L^{2}(\Omega, \mu)$. Since 1 and $h$ are linearly independent linear combinations of $\chi_{\Omega(+)}$ and $\chi_{\Omega(-)}$, these two indicator functions must both belong to $K$. We have

$$
\pi\left(u_{i}\right) 1=a_{i} \chi_{\Omega(-)}+\frac{1}{a_{i}} \chi_{\Omega\left(u_{i}\right)}-t a_{i} \sum_{j \neq i} \chi_{\Omega\left(u_{j}\right)},
$$

so $\left(1 / a_{i}\right) \chi_{\Omega\left(u_{i}\right)}-t a_{i} \sum_{j \neq i} \chi_{\Omega\left(u_{j}\right)} \in K$ for each $i$. It will follow that each $\chi_{\Omega\left(u_{i}\right)} \in K$ once we show that the matrix

$$
\left(\begin{array}{cccc}
1 / a_{1} & -t a_{1} & \cdots & -t a_{1} \\
-t a_{2} & 1 / a_{2} & \cdots & -t a_{2} \\
\vdots & \vdots & \ddots & \vdots \\
-t a_{n} & -t a_{n} & \cdots & 1 / a_{n}
\end{array}\right)
$$

is invertible. Multiplying row $i$ by $a_{i}\left(1+y_{i}\right)$ for each $i$ and using $t a_{i}^{2}=x_{i} /\left(1+y_{i}\right)$ turns this into

$$
\left(\begin{array}{cccc}
1+y_{1} & -x_{1} & \cdots & -x_{1} \\
-x_{2} & 1+y_{2} & \cdots & -x_{2} \\
\vdots & \vdots & \ddots & \vdots \\
-x_{n} & -x_{n} & \cdots & 1+y_{n}
\end{array}\right)
$$

whose determinant by Lemma 6.1 in the Appendix is

$$
(1+t)^{n}-(1+t)^{n-1} \sum_{j} x_{j}=(1+t)^{n-1} .
$$


We conclude that $\chi_{\Omega\left(u_{i}\right)} \in K$ for each $i$. Since

$$
\left(\pi\left(u_{i}^{-1}\right) \chi_{\Omega\left(u_{i}\right)}\right)(\omega)=P\left(u_{i}^{-1}, \omega\right) \chi_{\Omega\left(u_{i}\right)}\left(u_{i} \omega\right)= \begin{cases}0, & \text { if } p_{1}(\omega)=u_{i}^{-1} \\ a_{i}, & \text { otherwise }\end{cases}
$$

and since $K$ is $\pi(G)$-invariant, we see that $\chi_{\Omega\left(u_{i}^{-1}\right)}=1-\left(1 / a_{i}\right) \pi\left(u_{i}^{-1}\right) \chi_{\Omega\left(u_{i}\right)} \in K$. Suppose we have shown for some $k \geq 1$ that $\chi_{\Omega(s)} \in K$ for all reduced words $s$ of length $k$. For such a word $s$ not beginning with $u_{i}^{-1}$, we have

$$
\pi\left(u_{i}\right) \chi_{\Omega(s)}=P\left(u_{i}, \cdot\right) \chi_{\Omega\left(u_{i} s\right)}=\left(1 / a_{i}\right) \chi_{\Omega\left(u_{i} s\right)},
$$

so $\chi_{\Omega\left(u_{i} s\right)} \in K$. Likewise, if $s$ does not begin with $u_{i}$, then

$$
\pi\left(u_{i}^{-1}\right) \chi_{\Omega(s)}=P\left(u_{i}^{-1}, \cdot\right) \chi_{\Omega\left(u_{i}^{-1} s\right)}=c \chi_{\Omega\left(u_{i}^{-1} s\right)},
$$

where $c=1 / a_{i}$ if $s$ begins with a generator inverse, and $c=-1 /\left(t a_{i}\right)$ if $s$ begins with a generator. We conclude that the indicator function of every cylinder set belongs to $K$, showing that $K=L^{2}(\Omega, \mu)$. This takes care of (a) and (b).

To prove (c), it will suffice to show that $\sum_{i} c_{i} u_{i}^{-1}$ cannot have $\lambda$ as an eigenvalue in $\pi_{1}$. We will think of $\pi_{1}$ as acting on the Hilbert space

$$
H=H_{0} \oplus \bigoplus_{i=1}^{n}\left(\ell^{2}\left(S_{i}^{-}\right) \otimes H_{i}^{\prime}\right)
$$

constructed in Section 3 with $a_{i}$ and $b_{i j}$ as in Proposition 4.4. Thus, $H_{0}$ is the closed linear span of $\pi_{1}\left(G^{+}\right) \Delta_{1}$, while $H_{i}^{\prime}$ is $H_{0} \ominus \pi_{1}\left(u_{i}\right) H_{0}$, and so forth. Suppose now that $\xi$ in $H$ satisfies $\sum_{i} c_{i} \pi_{1}\left(u_{i}\right)^{*} \xi=\lambda \xi$. We claim first that $\xi$ must be orthogonal to each subspace $\ell^{2}\left(S_{j}^{-}\right) \otimes H_{j}^{\prime}$. Fix $j$, and $\eta$ in $H_{j}^{\prime}$. Define $f$ in $\ell^{2}(G)$ by

$$
f(s)= \begin{cases}\left\langle\xi, \delta_{s} \otimes \eta\right\rangle, & s \in S_{j}^{-} \\ 0, & \text { otherwise. }\end{cases}
$$

Notice that $\lambda\left\langle\xi, \delta_{s} \otimes \eta\right\rangle=\sum_{i} c_{i}\left\langle\xi, \pi\left(u_{i}\right)\left(\delta_{s} \otimes \eta\right)\right\rangle$ for every $s$ in $G$. We obtain

$$
\lambda f(s)-\sum_{i} c_{i} f\left(u_{i} s\right)= \begin{cases}c_{j}\langle\xi, \eta\rangle, & s=u_{j}^{-1} \\ 0, & \text { otherwise }\end{cases}
$$

by checking the cases $s \in S_{j}^{-} \backslash\left\{u_{j}^{-1}\right\}$ (use $\pi_{1}\left(u_{j}\right) \delta_{s} \otimes \eta=\delta_{u_{j} s} \otimes \eta$ ), $s=u_{j}^{-1}$ (use $\pi_{1}\left(u_{j}\right) \delta_{u_{j}^{-1}} \otimes \eta=\eta$ and $f(1)=0$ ), and $s \notin S_{j}^{-}$separately. This means that

$$
\left(\lambda-\sum_{i} c_{i} u_{i}^{-1}\right) *\left(f * u_{j}\right)=c_{j}\langle\xi, \eta\rangle \delta_{1} .
$$


The assumption is ambient that $\lambda-\sum c_{i} u_{i}$ (and hence its adjoint) is not invertible in the left regular representation, so $\langle\xi, \eta\rangle=0$ by Lemma 5.3 , and thus $f=0$ by Theorem 2.1 .

We have so far shown that $\xi \in H_{0}$, and that $\xi \perp H_{j}^{\prime}$ (that is, $\left.\xi \in \pi_{1}\left(u_{j}\right) H_{0}\right)$ for each $j$. Take $i \neq j$, for instance $i=1$ and $j=2$. Using part (c) of Lemma 3.3, we obtain $\xi=\left\langle\xi, \Delta_{u_{i}}\right\rangle \Delta_{u_{i}}=\left\langle\xi, \Delta_{u_{j}}\right\rangle \Delta_{u_{j}}$. This forces $\xi=0$, because $\Delta_{u_{i}}$ cannot be a scalar multiple of $\Delta_{u j}$. (The scalar in question would have to have modulus 1 because these are unit vectors, and would have to be $b_{i j}$ to make the inner product with $\Delta_{u_{j}}$ come out right. However, $\left|b_{i j}\right|<1$ because $b_{i j}$ is given by the formula in the statement of Proposition 4.4.)

A reasonable guess about the irreducible representations of $G$ treated in Section 4 is that they are classified up to unitary equivalence by the vector $\vec{c} / \lambda$. We leave this unresolved for now except to note that if one fixes $\vec{c}$ and changes $\lambda$, the new representation is inequivalent to the original one. This is an immediate consequence of the following proposition.

PROPOSITION 5.5. The only eigenvalue of $\sum c_{i} \pi_{1}\left(u_{i}\right)$ is $\lambda$. The corresponding eigenspace is $\mathbb{C} \Delta_{1}$.

PROOF. We use the notation of the proof of part (c) of the previous proposition. Suppose $\sum_{i} c_{i} \pi_{1}\left(u_{i}\right) \xi=\nu \xi$ for some complex number $\nu$ and some nonzero $\xi$ in $H$. Fix an index $j$, pick $\eta$ in $H_{j}^{\prime}$ and consider $g$ in $\ell^{2}(G)$ defined by

$$
g(s)= \begin{cases}\left\langle\xi, \delta_{s} \otimes \eta\right\rangle, & s \in S_{j}^{-} \\ 0, & \text { otherwise }\end{cases}
$$

One checks readily that $\left(\sum_{i} c_{i} u_{i}-v\right) * g=c_{j} g\left(u_{j}^{-1}\right) \delta_{1}$. Since $v$ belongs to the reduced spectrum of $\sum_{i} c_{i} u_{i}$, it follows from Lemma 5.3 that $g\left(u_{j}^{-1}\right)=0$, so $g$ vanishes identically by Theorem 2.1 . We have shown that $\xi$ must belong to $H_{0}$. By Lemma 3.3, then, we have

$$
v\left\langle\xi, \Delta_{1}\right\rangle=\sum_{i} c_{i}\left\langle\xi, \pi_{1}\left(U_{i}\right)^{*} \Delta_{1}\right\rangle=\sum_{i} c_{i} a_{i}\left\langle\xi, \Delta_{1}\right\rangle=\lambda\left\langle\xi, \Delta_{1}\right\rangle,
$$

and similarly for $s$ in $G^{+}$and any index $j$

$$
v\left\langle\xi, \Delta_{u_{j}}\right\rangle=c_{j}\left\langle\xi, \Delta_{s}\right\rangle+\phi(s)\left(\sum_{i \neq j} c_{i} b_{i j}\right)\left\langle\xi, \Delta_{1}\right\rangle .
$$

We can't have $\left\langle\xi, \Delta_{1}\right\rangle=0$, because that would force $\left\langle\xi, \Delta_{s}\right\rangle=0$ for all $s$ in $G^{+}$ and hence $\xi=0$. Hence $v=\lambda$. We may assume that $\left\langle\xi, \Delta_{1}\right\rangle=1$. The preceding formula becomes $\lambda\left\langle\xi, \Delta_{u_{j} s}\right\rangle=c_{j}\left\langle\xi, \Delta_{s}\right\rangle+\phi(s)\left(\lambda a_{j}-c_{j}\right)$, whence it readily follows 
by induction on the length of $s$ that $\left\langle\xi, \Delta_{s}\right\rangle=\phi(s)$ for every $s$ in $G^{+}$, and thus that $\xi=\Delta_{s}$.

We have so far avoided spectral values on the boundary of the reduced spectrum. The situation there is simpler and more clear-cut than in the interior (as well as being qualitatively different in the sense of [KS2]).

THEOREM 5.6. Let $\lambda=\left(\sum_{i} c_{i}^{2}\right)^{1 / 2}$, where $c_{1}, \ldots, c_{n}$ are nonnegative and not all zero. The function $\phi$ defined on $G$ by

$$
\phi(s)= \begin{cases}\prod_{i}\left(c_{i} / \lambda\right)^{|s|_{i}}, & \text { if } \gamma(s)=0 \\ 0, & \text { otherwise. }\end{cases}
$$

where we understand $0^{0}=1$, is the unique reduced $\lambda$-eigenstate for $\sum_{i} c_{i} u_{i}$.

PROOF. If only one of the coefficients is nonzero, this is Lemma 4.4 in [P]. Assume therefore that at least two coefficients are nonzero. The argument from [P] for the case $c_{1}=\cdots=c_{n}$ works here with just a few cosmetic changes.

Let $T=\lambda^{-1} \sum_{i} c_{i} u_{i}$, thought of as an operator on $\ell^{2}(G)$. Because 1 belongs to the reduced spectrum of $T$, there is a state $f$ on the algebra of bounded operators on $\ell^{2}(G)$ such that $f\left(\left(T^{*}-1\right)(T-1)\right)=0$. We will be done once we show that the restriction of $f$ to $G$ must coincide with $\phi$. Let $S^{+}$be the set of reduced words in $G$ beginning with some $u_{j}$, and let $S^{-}=G \backslash S^{+}$. Let $P$ and $Q$ be respectively the orthogonal projections of $\ell^{2}(G)$ on $\ell^{2}\left(S^{+}\right)$and $\ell^{2}\left(S^{-}\right)$, so $Q=1-P$. Suppose we know that $f(P)=1$. For an $s$ in $G$ with $\gamma(s)>0$ (that is, for $s$ not in $G^{+}\left(G^{+}\right)^{-1}$ ), we have $P\left(T^{*}\right)^{m} s T^{m} P=0$ for sufficiently large $m$, and hence $f(s)=f\left(P\left(T^{*}\right)^{m} s T^{m} P\right)=0$. On the other hand, if $s \in G^{+}\left(G^{+}\right)^{-1}$ and $s u_{j}^{-1}$ is reduced, then

$$
f\left(s u_{j}^{-1}\right)=f\left(s u_{j}^{-1} T\right)=\left(c_{j} / \lambda\right) f(s)
$$

because $\gamma\left(s u_{j}^{-1} u_{i}\right)=0$ for $i \neq j$. Likewise $f\left(u_{j} s\right)=\left(c_{j} / \lambda\right) f(s)$ if $u_{j} s$ is reduced. It now follows easily that $f(s)=\phi(s)$ for $s$ in as well as outside of $G^{+}\left(G^{+}\right)^{-1}$.

We show now that $f(P)$ must be 1 . Suppose not, that is, suppose $f(Q)>0$. Consider the state $g$ defined on bounded operators $X$ by $g(X)=f(Q X Q) / f(Q)$, so $g(Q)=1$. We have $Q T T^{*} Q=Q, Q T^{*} Q=T^{*} Q$, and $Q T Q=Q T$. Because $T$ is in the left kernel of $f$, this makes $g\left((T-1)\left(T^{*}-1\right)\right)=0$. The same argument as in the previous paragraph, mirror-imaged by the automorphism $\sigma$ of $G$ that sends each $u_{i}$ to $u_{i}^{-1}$, shows that $g=\phi \circ \sigma$. In particular, we have $g\left(u_{i}^{-1} u_{j}\right)=\lambda^{-2} c_{i} c_{j}$ for $i \neq j$. Consider now $f\left(u_{i}^{-1} u_{j}\right)$. We have already observed that

$$
f\left((Q-Q T)\left(Q-T^{*} Q\right)\right)=f(Q) g\left((T-1)\left(T^{*}-1\right)\right)=0,
$$


so $f\left(P u_{i}^{-1} u_{j} Q\right)=f\left(P u_{i}^{-1} u_{j} T^{*} Q\right)=f(0)=0$, and likewise $f\left(Q u_{i}^{-1} u_{j} P\right)=0$. Since also $P u_{i}^{-1} u_{j} P=0$, it follows that

$$
f\left(u_{i}^{-1} u_{j}\right)=f\left(Q u_{i}^{-1} u_{j} Q\right)=f(Q) g\left(u_{i}^{-1} u_{j}\right)=f(Q) \lambda^{-2} c_{i} c_{j}
$$

for $i \neq j$. All of these quantities are nonnegative, and at least two are positive. This, however, contradicts $f\left(T^{*} T\right)=f(1)=1$, because the latter forces the sum over unequal $i$ and $j$ of $c_{i} c_{j} f\left(u_{i}^{-1} u_{j}\right)$ to vanish.

Rotating the generators gets the uniqueness assertion above (with appropriately modified state formula) for an arbitrary nonzero coefficient vector $\vec{c}$ and complex $\lambda$ with $|\lambda|=|\vec{c}|$. As for the inner boundary of the spectral annulus, if $\left|c_{j}\right|$ is the maximum of the absolute coefficients, and if

$$
\left|c_{j}\right|^{2}>\sum_{i \neq j}\left|c_{i}\right|^{2} \text { and }|\lambda|^{2}=\left|c_{j}\right|^{2}-\sum_{i \neq j}\left|c_{i}\right|^{2}
$$

then $\sum_{i} c_{i} u_{i}$ has a unique reduced $\lambda$-eigenstate because $\lambda u_{j}^{-1}-\sum_{i \neq j} c_{i} u_{j}^{-1} u_{i}$ has a unique reduced $c_{j}$-eigenstate.

\section{Appendix. The map $S$}

Let $\mathbb{R}_{+}^{n}$ denote the positive orthant of $\mathbb{R}^{n}$. In this section we study the map $S: \mathbb{R}_{+}^{n} \rightarrow \mathbb{R}_{+}^{n}$ defined by

$$
S\left(x_{1}, x_{2}, \ldots x_{n}\right)=(1 / t)\left(x_{1}\left(1+y_{1}\right), x_{2}\left(1+y_{2}\right), \ldots, x_{n}\left(1+y_{n}\right)\right),
$$

where $t=\sum_{i} x_{i}$ and for each $j, y_{j}=\sum_{i \neq j} x_{i}$. We will use this notation- $t$ for the sum of the $x$ 's, $y_{j}$ for $t-x_{j}$-throughout our discussion of $S$. Our goal is to show that $S$ is one-to-one on $\mathbb{R}_{+}^{n}$ and that the range of $S$ is the open subset $D_{n}$ of $\mathbb{R}_{+}^{n}$ consisting of the points $\left(s_{1}, s_{2}, \ldots s_{n}\right)$ in $\mathbb{R}_{+}^{n}$ such that $\sum_{i} s_{i}>1$, and for each $j, s_{j}<1+\sum_{i \neq j} s_{i}$. That $S\left(\mathbb{R}_{+}^{n}\right) \subseteq D_{n}$ is elementary. If $s_{i}=x_{i}\left(1+y_{i}\right) / t$ for each $i$, then

$$
\sum_{i} s_{i}>\frac{1}{t} \sum_{i} x_{i}=1
$$

and for each $j$,

$$
\begin{aligned}
t\left(\sum_{i \neq j} s_{i}+1-s_{j}\right) & =\sum_{i \neq j} x_{i}\left(1+t-x_{i}\right)+t-x_{j}\left(1+t-x_{j}\right) \\
& =\left(t-x_{j}\right)(1+t)-\sum_{i \neq j} x_{i}^{2}+t-x_{j}-t x_{j}+x_{j}^{2}
\end{aligned}
$$




$$
\begin{aligned}
& =2\left(t-x_{j}\right)+\left(t-x_{j}\right)^{2}-\sum_{i \neq j} x_{i}^{2} \\
& =2 \sum_{i \neq j} x_{i}+\left(\sum_{i \neq j} x_{i}\right)^{2}-\sum_{i \neq j} x_{i}^{2}>0 .
\end{aligned}
$$

The derivative of $S$ is easily calculated. For $i \neq j$, the $(i, j)$ entry is given by

$$
S_{i, j}^{\prime}=\frac{\partial}{\partial x_{j}} \frac{x_{i}\left(1+y_{i}\right)}{t}=\frac{x_{i}^{2}-x_{i}}{t^{2}}
$$

while the diagonal entries are given by $S_{i, i}^{\prime}=\left(y_{i}^{2}+y_{i}\right) / t^{2}$. The following lemma will help show that det $S^{\prime}$ is positive on $\mathbb{R}_{+}^{n}$.

LEMMA 6.1. Let $M$ be an $n \times n$ matrix of the form

$$
\left(\begin{array}{cccc}
r_{1} & p_{1} & \cdots & p_{1} \\
p_{2} & r_{2} & \cdots & p_{2} \\
\vdots & \vdots & \ddots & \vdots \\
p_{n} & p_{n} & \cdots & r_{n}
\end{array}\right)
$$

Let $q_{j}=r_{j}-p_{j}$ for each $j$. The determinant of $M$ is $\prod_{i} q_{i}+\sum_{j} p_{j} \prod_{i \neq j} q_{i}$.

PROOF. Subtract the first column of $M$ from the other columns to obtain

$$
\begin{aligned}
\operatorname{det} M= & \left(r_{1} q_{2} q_{3} q_{4} \cdots q_{n}\right)+\left(p_{2} q_{1} q_{3} q_{4} \cdots q_{n}\right)+\left(p_{3} q_{2} q_{1} q_{4} \cdots q_{n}\right) \\
& +\cdots+\left(p_{n} q_{2} q_{3} q_{4} \cdots q_{n-1} q_{1}\right)
\end{aligned}
$$

then put $q_{1}+p_{1}$ for $r_{1}$ in the first term.

LEMMA 6.2. $\operatorname{det}\left(S^{\prime}\right)>0$ on $\mathbb{R}_{+}^{n}$.

PROOF. Notice that

$$
y_{j}^{2}+y_{j}-\left(x_{j}^{2}-x_{j}\right)=\left(t-x_{j}\right)^{2}-x_{j}^{2}+t=t\left(1+t-2 x_{j}\right)=t\left(1+y_{j}-x_{j}\right) .
$$

Let $\theta_{j}=1+y_{j}-x_{j}$. Apply the lemma above to $\operatorname{det}\left(t^{2} S^{\prime}\right)$ and then divide by $t^{n-1}$ to write

$$
\begin{aligned}
t^{n+1} \operatorname{det}\left(S^{\prime}\right) \equiv & P\left(x_{1}, x_{2}, \ldots, x_{n}\right) \\
= & \left(t \theta_{1} \theta_{2} \theta_{3} \cdots \theta_{n}\right)+\left(x_{1}^{2}-x_{1}\right)\left(\theta_{2} \theta_{3} \cdots \theta_{n}\right)+\theta_{1}\left(x_{2}^{2}-x_{2}\right)\left(\theta_{3} \cdots \theta_{n}\right) \\
& +\cdots+\left(\theta_{1} \theta_{2} \cdots \theta_{n-1}\right)\left(x_{n}^{2}-x_{n}\right) .
\end{aligned}
$$


Write the first term as $\sum_{j}\left(x_{j} \theta_{1} \theta_{2} \cdots \theta_{n}\right)$ and add the $j$ th term of this sum to the $j$ th of the remaining terms of $P$ to obtain

$$
P=\sum_{j}\left(x_{j} \theta_{j}+x_{j}^{2}-x_{j}\right) \prod_{i \neq j} \theta_{i}=\sum_{j} x_{j} y_{j} \prod_{i \neq j} \theta_{i}
$$

If all of the $\theta$ 's are positive, we conclude immediately that $P>0$. Otherwise, since at most one $x_{i}$ can exceed the sum of the others, all but one of the $\theta$ 's must be positive. Assume for definiteness that $\theta_{i}>0$ for $i \geq 2$. Writing $y_{1}$ as $x_{2}+\cdots+x_{n}$, we have

$$
P=\sum_{j \geq 2} x_{1} x_{j} \prod_{i \geq 2} \theta_{i}+\sum_{j \geq 2} x_{j} y_{j} \prod_{i \neq j} \theta_{i}=\sum_{j \geq 2} x_{j}\left(x_{1} \theta_{j}+y_{j} \theta_{1}\right) \prod_{i \neq 1, j} \theta_{i} .
$$

Now $x_{1} \theta_{j}+y_{j} \theta_{1}=x_{1}+y_{j}+y_{1} y_{j}-x_{1} x_{j}$, which is positive for $j \geq 2$ because $y_{1}>x_{j}$ and $y_{j}>x_{1}$.

Recall the open set $D_{n}$ defined at the beginning of this section.

LEMMA 6.3. If $\vec{\sigma}=\left(\sigma_{1}, \sigma_{2}, \ldots, \sigma_{n}\right)$ is the limit of a sequence $\left\{S\left(\vec{x}^{(m)}\right)\right\}$, where $\left\{\vec{x}^{(m)}\right\}_{m}$ is a sequence in $\mathbb{R}_{+}^{n}$ with no limit points in $\mathbb{R}_{+}^{n}$, then $\vec{\sigma} \notin D_{n}$.

PROOF. Omit the superscript $m$ and write

$$
s_{j}=\frac{x_{j}\left(1+y_{j}\right)}{x_{j}+y_{j}}, s_{j} \rightarrow \sigma_{j} \quad(j=1, \ldots, n) .
$$

After passing to a subsequence, we may assume that either $x_{j} \rightarrow 0$ for some $j$, or $x_{j} \rightarrow \infty$ for some $j$. Suppose that $x_{j} \rightarrow 0$ for some $j$. Notice that this forces $1>s_{j}>x_{j}$ eventually along the sequence. If $\sigma_{j}=0$, then $\vec{\sigma} \notin D_{n}$, and we are done. If $\sigma_{j}>0$, we may write

$$
y_{j}=\frac{x_{j}\left(s_{j}-1\right)}{x_{j}-s_{j}}
$$

and conclude that $y_{j} \rightarrow 0$. This makes $x_{i} \rightarrow 0$ for every $i$, and thus $y_{i} \rightarrow 0$ for every $i$. Since

$$
\frac{x_{i}}{x_{i}+y_{i}}+y_{i} \frac{x_{i}}{x_{i}+y_{i}} \rightarrow \sigma_{i}
$$

we must have $x_{i} /\left(x_{i}+y_{i}\right) \rightarrow \sigma_{i}$ for every $i$. Since $\sum_{i} x_{i} /\left(x_{i}+y_{i}\right)=1$, it follows that $\sum_{i} \sigma_{i}=1$, so $\vec{\sigma} \notin D_{n}$. The remaining possibility is that $x_{j} \rightarrow \infty$ for some $j$. This makes $1<s_{j}<x_{j}$ eventually along the sequence, and from

$$
y_{j}=\frac{s_{j}-1}{1-s_{j} / x_{j}}
$$


it follows that $y_{j} \rightarrow \sigma_{j}-1$. Since $y_{i} \rightarrow \infty$ for $i \neq j$, we also have

$$
x_{i}=\frac{s_{i} y_{i}}{1+y_{i}-s_{i}} \rightarrow \sigma_{i}
$$

for $i \neq j$. But $y_{j}=\sum_{i \neq j} x_{i}$, so $\sigma_{j}-1=\sum_{i \neq j} \sigma_{i}$, which rules $\vec{\sigma}$ out of $D_{n}$.

The referee of this paper has pointed out that the bijectivity of $S: \mathbb{R}_{+}^{n} \rightarrow D_{n}$ follows readily, without any further explicit calculation, from the preceding two lemmas and some elementary facts in homotopy theory. (For the latter, see [G, Part I] or [Spa, Chapter 2].) We finish our treatment of $S$ by presenting the referee's argument, which replaces several pages of figuring in the original.

It follows immediately from Lemma 6.3 that $S^{-1}(K)$ is compact for every compact subset $K$ of $D_{n}$ (in other words that $S: \mathbb{R}_{+}^{n} \rightarrow D_{n}$ is a proper map), and that $S\left(\mathbb{R}_{+}^{n}\right.$ ) is a closed subset of $D_{n}$. Lemma 6.2 implies that the image of $S$ is open, so $S$ must be surjective. The referee kindly supplied the statement and proof of the following proposition.

PROPOSITION 6.4. A surjective proper local homeomorphism between locally compact Hausdorff spaces is a covering map.

Proof. Let the map be $S: X \rightarrow Y$. Given $y$ in $Y$, we must find an open set $V$ containing $y$ such that $S^{-1}(V)$ is the disjoint union of open subsets of $X$ each of which is mapped homeomorphically onto $V$ by $S$. The hypotheses on $S$ make $S^{-1}(\{y\})$ a compact set with no limit points, thus finite, say $S^{-1}(\{y\})=\left\{x_{1}, \ldots, x_{k}\right\}$. Let $W_{1}, \ldots, W_{k}$ be disjoint open sets such that $x_{j} \in W_{j}$ for each $j$, and the restriction of $S$ to each $W_{j}$ is a homeomorphism. Let $V_{0}=\bigcap_{j} S\left(W_{j}\right)$, and for each $j$, let $U_{j}=S^{-1}\left(V_{0}\right) \cap W_{j}$. Then the $U_{j}$ 's are disjoint, each $U_{j}$ contains the corresponding $x_{j}$, and $S$ maps each $U_{j}$ homeomorphically onto $V_{0}$.

The reason we are not finished at this point is that the $U_{j}$ 's may not exhaust $S^{-1}\left(V_{0}\right)$. To make the appropriate adjustment, let $V_{1}$ be an open set containing $y$ such that $\bar{V}_{1}$ is a compact subset of $V_{0}$, and consider $K=S^{-1}\left(\bar{V}_{1}\right) \backslash \bigcup_{j} U_{j}$, which is a compact subset of $X$ because $S$ is proper. Then $S(K)$ is closed, and misses $y$ because $K$ misses $S^{-1}(\{y\})$, so $V=V_{1} \backslash S(K)$ is an open set containing $y$. The open sets $S^{-1}(V) \cap U_{j}$ are disjoint. Each of these sets is mapped homeomorphically onto $V$ (because $V \subseteq V_{0}=S\left(U_{j}\right)$ for each $j$ ), and that their union is $S^{-1}(V)$ (because $S(x) \in V$ implies $\left.x \in S^{-1}\left(V_{1}\right) \backslash K \subseteq \bigcup U_{j}\right)$.

In the situation of the proposition and its proof, suppose furthermore that $X$ is pathconnected and $Y$ is simply connected. Fix $y$ in $Y$ and suppose that $x_{0}, x_{1} \in S^{-1}(\{y\})$. There is a path $p$ in $X$ joining $x_{0}$ to $x_{1}$. Its image $S(p)$ is a loop at $y$. Because covering maps have the homotopy lifting property, shrinking the loop $S(p)$ to $y$ through loops 
based at $y$ lifts to an end-point preserving homotopy from $p$ to a path that lies in the finite set $S^{-1}(\{y\})$. Thus $x_{0}=x_{1}$, showing that $S$ is injective.

We conclude in particular that the map $S$ considered in this section maps $\mathbb{R}_{+}^{n}$ homeomorphically onto $D_{n}$.

\section{References}

[FTS] A. Figà-Talamanca and T. Steger, 'Harmonic analysis for anisotropic random walks on homogeneous trees', Mem. Amer. Math. Soc. 110 (1994), 531.

[G] M. Greenberg, Lectures on algebraic topology (Benjamin, New York, 1966).

[H] U. Haagerup, 'An example of a non nuclear $C^{*}$-algebra which has the metric approximation property', Invent. Math. 50 (1979), 279-293.

[KS1] G. Kuhn and T. Steger, 'More irreducible boundary representations', Duke Math. J. 82 (1996), 381-436.

[KS2] - 'Monotony of certain free group representations', J. Funct. Analysis 179 (2001), 1-17.

[KS3] - 'Paschke's conjecture for endpoint anisotropic series representations of the free group', preprint.

[L] P. A. Linnell, 'Division rings and group von Neumann algebras', Forum Math. 5(1993), 561-576.

[P] W. L. Paschke, 'Pure eigenstates for the sum of generators of the free group', Pacific J. Math. 197 (2001), 151-171; arXiv:math.OA/9906158.

[PV] M. Pimsner and D. Voiculescu, 'K-groups of reduced crossed products by free groups', J. Operator Theory 8 (1982), 131-156.

[Spa] E. H. Spanier, Algebraic topology (McGraw-Hill, New York, 1966).

[Spi] J. Spielberg, 'Free-product groups, Cuntz-Krieger algebras, and covariant maps', Internat. J. Math. 2 (1991), 457-476.

Department of Mathematics

University of Kansas

405 Snow Hall

Lawrence, KS 66045-2142

USA

e-mail: paschke@math.ukans.edu 